\title{
Nonuniform average sampling and reconstruction of signals with finite rate of innovation
}

Qiyu Sun

University of Central Florida

Find similar works at: https://stars.library.ucf.edu/facultybib2000 University of Central Florida Libraries http://library.ucf.edu

This Article is brought to you for free and open access by the Faculty Bibliography at STARS. It has been accepted for inclusion in Faculty Bibliography 2000 s by an authorized administrator of STARS. For more information, please contactSTARS@ucf.edu.

\section{Recommended Citation}

Sun, Qiyu, "Nonuniform average sampling and reconstruction of signals with finite rate of innovation" (2007). Faculty Bibliography 2000s. 7700.

https://stars.library.ucf.edu/facultybib2000/7700

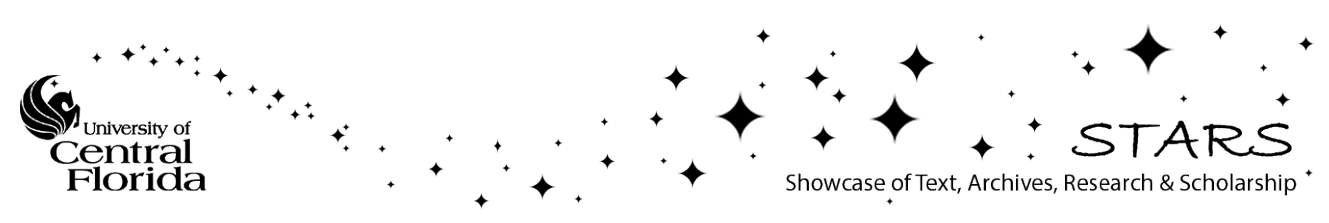




\title{
NONUNIFORM AVERAGE SAMPLING AND RECONSTRUCTION OF SIGNALS WITH FINITE RATE OF INNOVATION*
}

\author{
QIYU SUN ${ }^{\dagger}$
}

\begin{abstract}
From an average (ideal) sampling/reconstruction process, the question arises whether the original signal can be recovered from its average (ideal) samples and, if so, how. We consider the above question under the assumption that the original signal comes from a prototypical space modeling signals with a finite rate of innovation, which includes finitely generated shift-invariant spaces, twisted shift-invariant spaces associated with Gabor frames and Wilson bases, and spaces of polynomial splines with nonuniform knots as its special cases. We show that the displayer associated with an average (ideal) sampling/reconstruction process, which has a well-localized average sampler, can be found to be well-localized. We prove that the reconstruction process associated with an average (ideal) sampling process is robust, locally behaved, and finitely implementable, and thus we conclude that the original signal can be approximately recovered from its incomplete average (ideal) samples with noise in real time. Most of our results in this paper are new even for the special case when the original signal comes from a finitely generated shift-invariant space.
\end{abstract}

Key words. average sampling, ideal sampling, signals with finite rate of innovation, shiftinvariant spaces

AMS subject classifications. 42C15, 94A20, 46E30, 47B32, 41A15, $94 \mathrm{~A} 12$

DOI. $10.1137 / 05063444 \mathrm{X}$

1. Introduction. Modern digital data processing of functions (or signals or images) uses a discretized version of the original function that is obtained by (average) sampling on a discrete set [2]. The classical model is the Shannon sampling and reconstruction on the band-limited space $B_{\Omega}$, the space of all square-integrable functions on the real line with their Fourier transform supported in $[-\Omega, \Omega]$. From the Shannon sampling theorem, sampling a function $f$ in $B_{\pi}$ on the uniform grid $\mathbf{Z}$ yields an $\ell^{2}$ sequence $(f(k))_{k \in \mathbf{Z}}$, and conversely the original function $f$ can be recovered from its sampling data $\{f(k), k \in \mathbf{Z}\}$ by the following reconstruction formula:

$$
f(x)=\sum_{k \in \mathbf{Z}} f(k) \operatorname{sinc}(x-k), x \in \mathbf{R},
$$

where the sinc function is defined by $\operatorname{sinc}(x)=\frac{\sin \pi x}{\pi x}$. The above sampling and reconstruction theorem gives a framework for converting analogue signals into sequences, which can be processed digitally and converted back into analogue signals via the reconstruction formula (1.1). For the ideal sampling and reconstruction on the bandlimited space and the finitely generated shift-invariant spaces, there is an extensive literature (see, for example, the recent review papers [2, 57] and monographs [12, 14, 43]).

In most physical circumstances, due to the nonideal acquisition device at the sampling location, it is not realistic to measure the sample $f(\gamma)$ of the original signal $f$ in a space $V$ at the location $\gamma$ exactly. So a better assumption is that the sampled data are of the form $\left\langle f, \psi_{\gamma}\right\rangle$,

$$
A: V \ni f \longmapsto\left(\left\langle f, \psi_{\gamma}\right\rangle\right)_{\gamma \in \Gamma}
$$

*Received by the editors June 24, 2005; accepted for publication (in revised form) July 17, 2006; published electronically December 26, 2006.

http://www.siam.org/journals/sima/38-5/63444.html

${ }^{\dagger}$ Department of Mathematics, University of Central Florida, Orlando, FL 32816 (qsun@mail. ucf.edu). 
where $\psi_{\gamma}$, to be known as the average sampling functional, reflects the characteristic of the nonideal acquisition device at the sampling location $\gamma$. We call the above sampling process an average sampling process, and call the collection $\Psi:=\left\{\psi_{\gamma}, \gamma \in \Gamma\right\}$ of average sampling functionals an average sampler. Clearly, the average sampling process becomes an ideal sampling process if the delta function $\delta_{\gamma}$ is used as the average sampling functional $\psi_{\gamma}$ on every sampling location $\gamma$.

An easy model for the average sampling process is the discretization of the blurring process encountered in many practical situations, such as in the process of a remote camera imaging a scene and an observer viewing the sampled image [59]. For the average sampling and reconstruction on the band-limited space and on the finitely generated shift-invariant spaces, the reader may refer $[1,4,5,6,22,25,28,53,54,55$, $61]$ and references cited therein.

The question arises from the average sampling process whether the original function can be recovered from its average samples and, if so, how. Specifically, the first part of the above question, which will be discussed in section 4, can be described as follows: Given a class of functions $V$ on $\mathbf{R}^{d}$, find conditions on the average sampler $\Psi=\left\{\psi_{\gamma}: \gamma \in \Gamma\right\}$ under which any function $f$ in $V$ can be reconstructed uniquely and stably from its average samples $\left\{\left\langle f, \psi_{\gamma}\right\rangle: \gamma \in \Gamma\right\}$.

The second part of the above question arising from the average sampling process is the reconstruction process from the average (ideal) samples:

$$
D:\left(c_{\gamma}\right)_{\gamma \in \Gamma} \longmapsto \sum_{\gamma \in \Gamma} c_{\gamma} \tilde{\psi}_{\gamma} \in V
$$

such that

$$
D A f=f \text { for all } f \in V \text {. }
$$

Here for each $\gamma \in \Gamma$, the function $\tilde{\psi}_{\gamma}$, to be known as the display block at the location $\gamma$, reflects the characteristic of the display device at the sampling location $\gamma$. We call the above reconstruction process an average reconstruction process and the collection $\Psi:=\left\{\tilde{\psi}_{\gamma}, \gamma \in \Gamma\right\}$ of display blocks an displayer.

For the efficiency and stability of the reconstruction process (1.3), (1.4) to recover a function $f$ in the space $V$ from its averaging samples $\left\{\left\langle f, \psi_{\gamma}\right\rangle, \gamma \in \Gamma\right\}$ or from its ideal samples $\{f(\gamma), \gamma \in \Gamma\}$, we require the corresponding displayer $\tilde{\Psi}:=\left\{\tilde{\psi}_{\gamma}, \gamma \in \Gamma\right\}$ to be well-localized, and the average sampling/reconstruction process (1.3), (1.4) to be robust, local-behaved, and finitely implementable. In this paper, we show that those natural requirements for the average (ideal) sampling/reconstruction process would be met when signals in the space $V$ have a finite rate of innovation and the average sampler $\Psi$ is well-localized; see section 2.3 for our reasons for considering sampling/reconstruction of signals with a finite rate of innovation. Here a signal is said to have a finite rate of innovation if it has a finite degree of freedom per unit of time; see [23, 34, 40, 42, 45, 46, 58].

The paper is organized as follows. We divide section 2 into five subsections. In the first three subsections, we make some basic assumptions on the sampling set $\Gamma$, the average sampler $\Psi=\left\{\psi_{\gamma}, \gamma \in \Gamma\right\}$, and the space $V$, which is where the original function $f$ for the average sampling/reconstruction process comes from. Briefly, we assume that the sampling set $\Gamma$ is a relatively separated subset of $\mathbf{R}^{d}$, the average sampler $\Psi$ is well-localized in the sense that every average sampling functional $\psi_{\gamma}$ in the average sampler $\Psi$ is essentially located in a neighborhood of $\gamma \in \Gamma$, and the space $V$ is the space $V_{q}(\Phi, \Lambda)$, that is, as originally introduced in [52] for modeling 
signals with a finite rate of innovation. In the last two subsections, we recall some basic properties of the space $V_{q}(\Phi, \Lambda)$ from [52], and introduce a simplified model of our average (ideal) sampling/reconstruction process for the readers' convenience.

Since each display block $\tilde{\psi}_{\gamma}$ in the displayer $\tilde{\Psi}:=\left\{\tilde{\psi}_{\gamma}, \gamma \in \Gamma\right\}$ reflects the characteristic of the display device at the sampling location $\gamma, \gamma \in \Gamma$, it is reasonable to require that for each $\gamma \in \Gamma$, the display block $\tilde{\psi}_{\gamma}$ be essentially supported in a neighborhood of the sampling location $\gamma$. In section 3, derived from a general theorem for localized frames (see [8, Theorem 1], [24, Theorem 3.6], and [32, Theorem 13]), it is shown that such a requirement would be met for average (ideal) sampling in the space $V_{2}(\Phi, \Lambda)$ if the average sampler $\Psi$ and the generator $\Phi$ for the space $V_{2}(\Phi, \Lambda)$ are well-localized (Theorems 3.1 and 3.2); see Remark 3.1 for a more general formulation of the well-localization of a displayer. The well-localization of displayers will play a crucial role in our study of stable average sampling in $V_{r}(\Phi, \Lambda)$ with $r \neq 2$ (Corollary 3.4), the robustness and local convergence of the reconstruction process from average (ideal) samples (Theorems 5.1-5.3 and 6.1-6.3), and exponential convergence of an iterative algorithm for the reconstruction process from average (ideal) samples (Theorems 7.1 and 7.2).

In section 4 , we find conditions on the average sampling sampler $\Psi=\left\{\psi_{\gamma}: \gamma \in \Gamma\right\}$ (respectively, on the ideal sampling set $\Gamma$ ) under which any function $f$ in $V_{2}(\Phi, \Lambda)$ can be reconstructed uniquely and stably from its average samples $\left\{\left\langle f, \psi_{\gamma}\right\rangle: \gamma \in \Gamma\right\}$ (respectively, from its ideal samples $\{f(\gamma), \gamma \in \Gamma\}$ ); see Theorems 4.1 and 4.2.

In the average (ideal) sampling/reconstruction process, we should bring the following situations into our consideration: the average samples $\left\{\left\langle f, \psi_{\gamma}\right\rangle: \gamma \in \Gamma\right\}$ may involve some noises (caused by, for example, measurement, storage, or transmission), and the average sampler $\Psi$ may not be exactly the same as the one we expect (because of the mathematical modeling or the measurement of the acquisition device). In section 5 , we consider the numerical stability of the reconstruction process (1.3), (1.4). We show that if the average sampler $\Psi$ and the generator $\Phi$ for the space $V_{2}(\Phi, \Lambda)$ are well-localized, then the reconstruction process (1.3), (1.4) for $f \in V_{2}(\Phi, \Lambda)$ is stable under the corruption of the average (ideal) sampling data, and under the perturbation of averaging samplers, ideal sampling sets and the displayers; see Theorems 5.1, 5.2, and 5.3 for details. Then we conclude that the reconstruction process (1.3), (1.4) for $f \in V_{2}(\Phi, \Lambda)$ is robust.

By the reconstruction process (1.3), (1.4), any function $f$ in the space $V$ can be recovered fully when its average (ideal) sampling data are received completely. In some situations (such as when data missing are in the transmission and in the real-time reconstruction process), we are required to recover the original function (signal) partially from incomplete (ideal) average samples. We observe from the well-localization of the average sampler $\Psi$ and of the ideal sampling set $\Gamma$ that the average sampling data $\left\langle f, \psi_{\gamma}\right\rangle$ and the ideal sampling data $f(\gamma)$ catch the information of the function $f$ essentially in a neighborhood of the sampling location $\gamma$ for every $\gamma \in \Gamma$, which implies that the average (ideal) sampling procedure is locally behaved. So a natural question is whether the reconstruction procedure is locally behaved, or particularly whether a function $f \in V_{2}(\Phi, \Lambda)$ on a certain region $K$ can be recovered approximately (or exactly) from the average sampling data $\left\langle f, \psi_{\gamma}\right\rangle$ and the ideal sampling data $f(\gamma)$ for the sampling location $\gamma$ in a neighborhood of that region. In section 6 , it is proved that for any bounded region $K$, the original function in the space $V_{2}(\Phi, \Lambda)$ can be approximately recovered from its average (ideal) samples in an $R$-neighborhood $B(K, R)=\left\{y: \inf _{x \in K}|y-x| \leq R\right\}$ of that region $K$ via a finite algorithm (see 
Theorems 6.1 and 6.2 for details), and moreover that the local convergence rate of the local reconstruction procedure is almost the same as the rate of polynomial (subexponential) decay of the generator $\Phi$ and of the average sampler $\Psi$. Therefore we conclude that the reconstruction process (1.3), (1.4) for $f \in V_{2}(\Phi, \Lambda)$ is locally behaved and finitely implementable (hence it could lead possibly to a real-time reconstruction algorithm) when the average sampler $\Psi$ and the generator $\Phi$ are well-localized. As a by-product of the local reconstruction theorems, we obtain a necessary condition on the location $\Gamma$ of average (ideal) sampling devices, which states that, for a stable average (ideal) sampling/reconstruction procedure on the space $V_{2}(\Phi, \Lambda)$, there exists a positive constant $R_{0}$ such that for any domain $K \subset \mathbf{R}^{d}$, the number of average (ideal) sampling devices located in $R_{0}$-neighborhood $B\left(K, R_{0}\right)$ of that domain $K$ should exceed the degrees of freedom of the space $V_{2}(\Phi, \Lambda)$ in the domain $K$; see Theorem 6.3 for details. The above necessary condition, which is usually known as the density property, is established in [3] for the ideal sampling on the B-spline space (see $[2,3]$ and references cited therein for nonuniform sampling on the band-limited space, and see $[8,9]$ for nonuniform Gabor system).

In the average (ideal) sampling/reconstruction process, we need an efficient and fast numerical algorithm that recovers any function $f \in V_{r}(\Phi, \Lambda)$ from its average sampling values $\left\langle f, \psi_{\gamma}\right\rangle, \gamma \in \Gamma$, or from its ideal sampling values $f(\gamma), \gamma \in \Gamma$. In section 7 , we modify the standard Richardson-Landweber iterative frame algorithm to implement the reconstruction process (1.3), (1.4) for signals $f \in V_{r}(\Phi, \Lambda)$ when average (ideal) samples are received completely, and show that the new iterative algorithm converges exponentially for any initial data in $\ell^{r}$ and that the limit agrees with the signal in the space $V_{r}(\Phi, \Lambda)$ whenever the initial data are obtained from average (ideal) sampling of that signal (see $[2,6,22]$ for convergence results similar to the standard Richardson-Landweber iterative frame algorithm in the shift-invariant setting). The Richardson-Landweber iterative algorithm is easily implemented, but it provides slow convergence in general. Relaxation and acceleration techniques, such as the conjugate gradient acceleration, help to alleviate the convergence problem [29,30], but their consideration is beyond the scope of this paper and will be discussed in a subsequent paper.

The proofs of all results are collected in section 8 .

In this paper, an uppercase letter $C$, if unspecified, denotes an absolute constant which may be different at different occurrences.

\section{Preliminaries.}

2.1. The sampling set $\boldsymbol{\Gamma}$. Every $\gamma$ in the sampling set $\Gamma$ is used as the location of a (non-)ideal sampling acquisition device, which has the average sampling characteristic $\psi_{\gamma}$. Then reasonable assumptions on the sampling set $\Gamma$ are that only finitely many such sampling acquisition devices are located in any unit interval, and that the distribution of those devices is almost location invariant. So in this paper, we make the following basic assumption on the sampling set $\Gamma$ :

(i) The sampling set $\Gamma$ is a relatively separated subset of $\mathbf{R}^{d}$.

Here, given a subset $X=\left\{x_{j}\right\}$ of $\mathbf{R}^{d}$, we say that $X$ is relatively separated if there exists a positive constant $D(X)$ such that

$$
\sum_{x_{j} \in X} \chi_{x_{j}+[0,1]^{d}}(x) \leq D(X) \quad \text { for all } x \in \mathbf{R}^{d} .
$$

2.2. The average sampler $\boldsymbol{\Psi}$. We say that a positive function $u$ on $\mathbf{R}^{d}$ is a weight if it is continuous, symmetric, and satisfies $1=u(0) \leq u(x)<\infty$ for 
all $x \in \mathbf{R}^{d}$, and the inequality $u(x+y) \leq u(x) v(y), x, y \in \mathbf{R}^{d}$, holds for another continuous function $v$ on $\mathbf{R}^{d}$. The model examples of weights convenient for our consideration of the sampling/reconstruction process are the polynomial weights

$$
u_{\alpha}(x)=(1+|x|)^{\alpha}
$$

with $\alpha \geq 0$, and the subexponential weights

$$
e_{D, \delta}(x)=\exp \left(D|x|^{\delta}\right)
$$

with $D>0$ and $\delta \in(0,1)$.

Given $1 \leq p, q \leq \infty$, a weight $u$, a relatively separated subset $\Gamma$ of $\mathbf{R}^{d}$, and a family $\Psi=\left\{\psi_{\gamma}: \gamma \in \Gamma\right\}$ of functions on $\mathbf{R}^{d}$, we define $\|\Psi\|_{q, p, u}$ by

$$
\begin{aligned}
\|\Psi\|_{q, p, u}:= & \sup _{\gamma \in \Gamma}\left\|\left(\left\|\psi_{\gamma}(\cdot) u(\cdot-\gamma)\right\|_{L^{q}\left(k+[0,1]^{d}\right)}\right)_{k \in \mathbf{Z}^{d}}\right\|_{\ell^{p}\left(\mathbf{Z}^{d}\right)} \\
& +\sup _{k \in \mathbf{Z}^{d}}\left\|\left(\left\|\psi_{\gamma}(\cdot) u(\cdot-\gamma)\right\|_{L^{q}\left(k+[0,1]^{d}\right)}\right)_{\gamma \in \Gamma}\right\|_{\ell^{p}(\Gamma)},
\end{aligned}
$$

where, as usual, $\|\cdot\|_{L^{q}(K)}$ denotes the usual $L^{q}$ norm on the space $L^{q}(K)$ of all $q$-integrable functions on a measurable set $K$, and $\|\cdot\|_{\ell^{p}(X)}$ (or $\|\cdot\|_{\ell^{p}}$ for short) is the usual $\ell^{p}(X)$ norm on the space of all $q$-summable sequences on the index set $X$. For $q=p=\infty$, it is obvious that $\|\Psi\|_{q, p, u}<\infty$ if and only if $\left|\psi_{\gamma}(x)\right| \leq$ $\|\Psi\|_{q, p, u}(u(x-\gamma))^{-1}$ for all $x \in \mathbf{R}^{d}$ and $\gamma \in \Gamma$. In general, for the family of functions $\Psi=\left\{\psi_{\gamma}: \gamma \in \Gamma\right\}$ with $\|\Psi\|_{q, p, u}<\infty$, each function $\psi_{\gamma}, \gamma \in \Gamma$, is an $L^{q}$ function "locally" and a weighted $L^{p}$ function centered at $\gamma$ "globally." Therefore for each $\gamma \in \Gamma$, the function $\psi_{\gamma}$ in the collection $\Psi:=\left\{\psi_{\gamma}, \gamma \in \Gamma\right\}$ with $\|\Psi\|_{q, p, u}<\infty$ can be thought of as essentially supported in a neighborhood of $\gamma \in \Gamma$.

For the average sampler $\Psi=\left\{\psi_{\gamma}: \gamma \in \Gamma\right\}$, each average sampling functional $\psi_{\gamma}$ reflects the characteristic of the nonideal acquisition device at the location $\gamma \in \Gamma$, and hence it should be essentially supported in a neighborhood of the sampling location $\gamma$. So we make the following basic assumption on the average sampler $\Psi$ :

(ii) The average sampler $\Psi=\left\{\psi_{\gamma}: \gamma \in \Gamma\right\}$ satisfies

$$
\|\Psi\|_{q, p, u}<\infty
$$

for some $1 \leq p, q \leq \infty$, and weight $u$.

We interpret any average sampler that satisfies the basic assumption (ii) as having polynomial (subexponential) decay, due to the interpretation of the collection $\Psi$ of average sampling functional $\psi_{\gamma}, \gamma \in \Gamma$, with $\|\Psi\|_{q, p, u}<\infty$ and the model assumption on the weight $u$ that is convenient for our consideration of the sampling/reconstruction process in which $u$ is a polynomial weight $u_{\alpha}$ or a subexponential weight $e_{D, \delta}$.

Remark 2.1. For adapting to different average (ideal) sampling situations, we add some flexibility to the basic assumption (ii) on the average sampler $\Psi$ with variable exponents $p$ and $q$ and weights $u$. For instance, we may use $q=1$ for approximating ideal sampling $\left(\psi_{\gamma} \approx \delta_{\gamma}\right.$; see [6]), $q=2$ for frame sampling (for instance, $\Psi=$ $\left\{\phi(\cdot-k), k \in \mathbf{Z}^{d}\right\}$ for frame sampling in the shift-invariant space $V_{2}(\phi)$ generated by $\phi,[7,13,39,52]), q=\infty$ in local blurring or local averaging (for instance, $\psi_{\gamma}=h(\cdot-\gamma)$ for some compactly supported phase function $h,[5,25,59])$, and the subexponential weight $e_{D, \delta}$ for oversampling band-limited signals [35].

Remark 2.2. Given $p \in[1, \infty]$, a weight $u$, and two relatively separated subsets $\Gamma, \Gamma^{\prime}$ of $\mathbf{R}^{d}$, we define the matrix algebra $\mathcal{A}_{p, u}\left(\Gamma, \Gamma^{\prime}\right)$ of Schur class by

$$
\mathcal{A}_{p, u}\left(\Gamma, \Gamma^{\prime}\right):=\left\{A:=\left(A\left(\gamma, \gamma^{\prime}\right)\right)_{\gamma \in \Gamma, \gamma^{\prime} \in \Gamma^{\prime}}:\|A\|_{\mathcal{A}_{p, u}}<\infty\right\},
$$


where

$$
\|A\|_{\mathcal{A}_{p, u}}:=\sup _{\gamma \in \Gamma}\left\|\left(A\left(\gamma, \gamma^{\prime}\right) u\left(\gamma-\gamma^{\prime}\right)\right)_{\gamma^{\prime} \in \Gamma^{\prime}}\right\|_{\ell^{p}\left(\Gamma^{\prime}\right)}+\sup _{\gamma^{\prime} \in \Gamma^{\prime}}\left\|\left(A\left(\gamma, \gamma^{\prime}\right) u\left(\gamma-\gamma^{\prime}\right)\right)_{\gamma \in \Gamma}\right\|_{\ell^{p}(\Gamma)}
$$

(see, e.g., $[32,37,51]$ ). Then the basic assumption $\|\Psi\|_{q, p, u}<\infty$ on the average sampler $\Psi$ is characterized by

$$
\|\Psi\|_{q, p, u}<\infty \text { if and only if }\left(\left\|\psi_{\gamma}\right\|_{L^{q}\left(k+[0,1]^{d}\right)}\right)_{\gamma \in \Gamma, k \in \mathbf{Z}^{d}} \in \mathcal{A}_{p, u}\left(\Gamma, \mathbf{Z}^{d}\right) .
$$

For the basic assumption $\|\Psi\|_{q, p, u}<\infty$ with different exponents $p, q$ and weights $u$, we have the following results, which will be used frequently in the proofs:

$$
\|\Psi\|_{q_{1}, p, u} \leq C\|\Psi\|_{q_{2}, p, u}
$$

if $q_{1} \leq q_{2}$, and

$$
\|\Psi\|_{q, p_{1}, u} \leq C\|\Psi\|_{q, p_{2}, v}
$$

if $p_{1} \leq p_{2}$ and $\left\|u v^{-1}\right\|_{L^{r}}<\infty$, where $1 / r=1 / p_{1}-1 / p_{2}$; see [52] for details.

2.3. The space $V$ in which functions are sampled and recovered. The band-limited space $B_{\Omega}, \Omega>0$, is a prototypical space for sampling theory and for signal processing in the classical band-limited model [2]. By the Whittaker representation theorem, the band-limited space $B_{\pi}$ is spanned by the shifted sinc function $\operatorname{sinc}(x-k):=\frac{\sin \pi(x-k)}{\pi(x-k)}, k \in \mathbf{Z}$, using $\ell^{2}$ coefficients, i.e., $B_{\Omega}=\left\{\sum_{k \in \mathbf{Z}} c(k) \operatorname{sinc}(x-k)\right.$ : $\left.(c(k)) \in \ell^{2}\right\}$.

Since the sinc function has infinite support and slow decay at infinity, the bandlimited space is often unsuitable for numerical implementations (see, e.g., [2, 35]). Hence people consider other models that retain some of the simplicity and structure of the band-limited model, but are more amenable to numerical implementation and are more flexible for approximate real data (see $[2,11,15,36,56]$ and references cited therein).

Other than the band-limited model, a widely used model is the finitely generated shift-invariant model; see, e.g., [1, 2, 4, 5, 6, 19, 28, 41, 53, 54, 55]. Here the finitely generated shift-invariant space $V_{q}\left(\phi_{1}, \ldots, \phi_{N}\right)$, that has functions $\phi_{1}, \ldots, \phi_{N}$ on $\mathbf{R}^{d}$ as its generators, is defined by

$$
V_{q}\left(\phi_{1}, \ldots, \phi_{N}\right):=\left\{\sum_{n=1}^{N} \sum_{k \in \mathbf{Z}^{d}} c_{n}(k) \phi_{n}(\cdot-k):\left(c_{n}(k)\right)_{k \in \mathbf{Z}^{d}} \in \ell^{q}, 1 \leq n \leq N\right\},
$$

where $1 \leq q \leq \infty$ (see, e.g., $[7,13,16,21,39]$ for the applications of finitely generated shift-invariant spaces in wavelet analysis and approximation theory). Clearly the finitely generated shift-invariant space $V_{q}\left(\phi_{1}, \ldots, \phi_{N}\right)$ becomes the band-limited space $B_{\pi}$ if we let $q=2, N=1$, and $\phi_{1}=$ sinc.

The space $V_{q}(\Phi, \Lambda)$,

$$
V_{q}(\Phi, \Lambda):=\left\{\sum_{\lambda \in \Lambda} c(\lambda) \phi_{\lambda}:\left\|(c(\lambda))_{\lambda \in \Lambda}\right\|_{\ell^{q}(\Lambda)}<\infty\right\},
$$

that was recently introduced by the author in [52] is a new model (other than the above band-limited model and shift-invariant model), where $1 \leq q \leq \infty, \Lambda$ is a relatively separated subset of $\mathbf{R}^{d}$, and $\Phi=\left\{\phi_{\lambda}, \lambda \in \Lambda\right\}$ satisfies $\|\Phi\|_{q, p, u}<\infty$ for 
some $1 \leq p, q \leq \infty$ and some weight $u$. We call $\Phi$ the generator of the space $V_{q}(\Phi, \Lambda)$, and call $\Lambda$ the location of the generator.

The prototypical space $V_{q}(\Phi, \Lambda)$ has shift-invariant spaces, twisted shift-invariant spaces generated by (non-)uniform Gabor frame system (or Wilson basis) in the timefrequency analysis (see, e.g., [8, 17, 26, 38, 47] and references cited therein), and spaces of polynomial splines (which are widely used as approximating spaces in data fitting problems and operator-equation problems $[15,36,48]$ ) as its special cases. Particularly the space $V_{q}(\Phi, \Lambda)$ and the shift-invariant space $V_{q}\left(\phi_{1}, \ldots, \phi_{N}\right)$ are related as follows:

$$
V_{q}(\Phi, \Lambda)=V_{q}\left(\phi_{1}, \ldots, \phi_{N}\right)
$$

and

$$
\|\Phi\|_{q, p, u}<\infty \text { if and only if } \phi_{1}, \ldots, \phi_{N} \in W_{q}\left(L_{p, u}\right)
$$

if we let $\Lambda:=\left\{x_{1}, \ldots, x_{N}\right\}+\mathbf{Z}^{d}$ and $\phi_{\lambda}:=\phi_{n}(\cdot-k)$ if $\lambda=x_{n}+k$ for some $k \in \mathbf{Z}^{d}$, where $\left\{x_{1}, \ldots, x_{N}\right\}$ is a discrete set in $\mathbf{R}^{d} / \mathbf{Z}^{d}$; see [52] for details. Here we recall that the Wiener amalgam space $W_{q}\left(L_{p, u}\right)$, which consists of functions that are "locally" in $L^{q}$ and "globally" in weighted $L^{p}$ space with weight $u$ [2], is defined by

$$
W_{q}\left(L_{p, u}\right):=\left\{f:\|f\|_{W_{q}\left(L_{p, u}\right)}:=\left\|\left(\|f u\|_{L^{q}\left(k+[0,1]^{d}\right)}\right)_{k \in \mathbf{Z}^{d}}\right\|_{\ell^{p}\left(\mathbf{Z}^{d}\right)}<\infty\right\} .
$$

The prototypical space $V_{q}(\Phi, \Lambda)$ is suitable for modeling signals with a finite rate of innovations $[23,34,40,42,44,45,46,58]$ in, for instance, (i) stream of pulses $\sum_{l} a_{l} p(t-$ $t_{l}$ ) found in global positioning system (GPS) applications and cellular radio, where $p(t)$ is the antenna transmit pulse shape; (ii) stream of different pulses $\sum_{l} a_{l} p_{l}\left(t-t_{l}\right)$ found in modeling ultra wide-band, where different incoming paths are subjected to different frequency-selective attenuations; (iii) band-limited signals with additive shot noise $\sum_{k \in \mathbf{Z}} c(k) \operatorname{sinc}(t-k)+\sum_{l} d(l) \delta\left(t-t_{l}\right)$; (iv) sum of band-limited signals and nonuniform spline signals, convenient for modeling electrocardiogram signals.

The prototypical space $V_{q}(\Phi, \Lambda)$ retains some of the simplicity and structure of a finitely generated shift-invariant space of the form (2.10), is amenable to numerical implementation (see sections 5,6, and 7), and is more flexible for approximating real data than the band-limited model and the shift-invariant model (see [52] for details).

So in this paper, we make the following basic assumption on the space $V$ in which functions are sampled and recovered:

(iii) The space $V$ is of the form $V_{q}(\Phi, \Lambda)$, where $1 \leq q \leq \infty, \Lambda$ is a relatively separated subset of $\mathbf{R}^{d}$, and $\Phi=\left\{\phi_{\lambda}: \lambda \in \Lambda\right\}$ is a family of functions on $\mathbf{R}^{d}$ satisfying $\|\Phi\|_{q, p, u}<\infty$ for some $1 \leq p \leq \infty$ and weight $u$.

Remark 2.3. Signals in the space $V:=V_{q}(\Phi, \Lambda)$, which satisfies the above basic assumption (iii), have a finite rate of innovation because a signal $f=\sum_{\lambda \in \Lambda} c(\lambda) \phi_{\lambda} \in$ $V_{q}(\Phi, \Lambda)$ on a unit interval $t+[-1 / 2,1 / 2)^{d}$ is essentially determined by the coefficients $c(\lambda)$ with $\lambda \in t+[-1 / 2,1 / 2)^{d}$ because of the well-localization property of the generator $\Phi$, and the total number of the locations $\lambda \in \Lambda$ on the unit interval $t+[-1 / 2,1 / 2)^{d}$ is bounded by some constant $C_{0}$ independent of the center $t$ of the unit interval due to the relative separatedness of the location $\Lambda$ of the generator $\Phi$.

Remark 2.4. We provide some flexibility on the assumption $\|\Phi\|_{q, p, u}<\infty$ on the generator $\Phi$ of the space $V_{q}(\Phi, \Lambda)$ for adapting to different modeling situations. For instance, we may use $q=1$ and $p=\infty$ when modeling slow-varying signals with shot noises [58], $1 \leq p, q \leq \infty$ when modeling signals in a finitely generated shift-invariant space [2], and $q=\infty$ and $1 \leq p \leq \infty$ for decomposing a time signal via (non-)uniform Gabor frame system or Wilson basis [26]. 
2.4. The space $V_{q}(\Phi, \Lambda)$ for modeling signals with a finite rate of innovations. Let $\delta_{\lambda \lambda^{\prime}}$ stand for the usual Kronecker symbol. For a Hilbert space $H$ with $E=\left\{e_{\lambda}, \lambda \in \Lambda\right\}$ being its Riesz basis, we say that $E^{d}=\left\{e_{\lambda}^{d}: \lambda \in \Lambda\right\} \subset H$ is a dual Riesz basis of $E$ if $E^{d}$ is a Riesz basis of $H$ and $\left\langle e_{\lambda}, e_{\lambda^{\prime}}^{d}\right\rangle=\delta_{\lambda \lambda^{\prime}}$ for all $\lambda, \lambda^{\prime} \in \Lambda$, and we say that $E^{o}=\left\{e_{\lambda}^{o}: \lambda \in \Lambda\right\}$ is an orthonormal basis for $H$ if $E^{o}$ is a basis of $H$ and $\left\langle e_{\lambda}^{o}, e_{\lambda^{\prime}}^{o}\right\rangle=\delta_{\lambda \lambda^{\prime}}$ for all $\lambda, \lambda^{\prime} \in \Lambda$.

Let $1 \leq p \leq \infty$. We say that a weight $u$ is $p$-admissible if there exist a weight $v$ and two positive constants $D:=D(u) \in(0, \infty)$ and $\theta:=\theta(u) \in(0,1)$ such that

$$
\begin{gathered}
u(x+y) \leq D(u(x) v(y)+v(x) u(y)) \quad \text { for all } x, y \in \mathbf{R}^{d}, \\
\left\|\left(v u^{-1}\right)\right\|_{L^{p^{\prime}}} \leq D, \text { and } \\
\inf _{\tau>0}\|v\|_{L^{1}(B(\tau))}+t\left\|v u^{-1}\right\|_{L^{p^{\prime}}\left(\mathbf{R}^{d} \backslash B(\tau)\right)} \leq D t^{\theta} \quad \text { for all } t \geq 1,
\end{gathered}
$$

where $p^{\prime}=p /(p-1)$ and $B(\tau)=\left\{x \in \mathbf{R}^{d}:|x| \leq \tau\right\}$. The $p$-admissibility of a weight $u$ is a technical condition in [51] for establishing the Wiener lemma for matrix algebras of Schur class and of Sjöstrand class; see also Lemmas 8.1 and 8.2. It is verified in [51] that the polynomial weight $u_{\alpha}$ with $\alpha>d(1-1 / p)$ and the subexponential weight $e_{D, \delta}$ with $D>0$ and $\delta \in(0,1)$ are $p$-admissible weights. The reader may refer to those two model examples for simplification; see also subsection 2.5.

Now we recall some properties of the space $V_{q}(\Phi, \Lambda)$ in [52]; see, e.g., $[2,39]$ for similar results for our familiar shift-invariant setting.

Proposition 2.1 (see [52]). Let $1 \leq q \leq \infty, u_{\alpha}(x):=(1+|x|)^{\alpha}, \alpha \geq 0$, be the polynomial weights, $\Lambda$ be a relatively separated subset of $\mathbf{R}^{d}, \Phi=\left\{\phi_{\lambda}, \lambda \in \Lambda\right\}$ satisfy $\|\Phi\|_{q, 1, u_{0}}<\infty$, and $V_{q}(\Phi, \Lambda)$ be defined as in (2.11). Then $V_{q}(\Phi, \Lambda) \subset L^{q}$. Moreover,

$$
\left\|\sum_{\lambda \in \Lambda} c(\lambda) \phi_{\lambda}\right\|_{L^{r}} \leq C\left\|(c(\lambda))_{\lambda \in \Lambda}\right\|_{\ell^{r}(\Lambda)}\|\Phi\|_{q, 1, u_{0}}
$$

for every sequence $(c(\lambda))_{\lambda \in \Lambda} \in \ell^{r}(\Lambda)$ with $1 \leq r \leq q$, and

$$
\left\|\left(\left\langle f, \phi_{\lambda}\right\rangle\right)_{\lambda \in \Lambda}\right\|_{\ell^{r}(\Lambda)} \leq C\|f\|_{L^{r}}\|\Phi\|_{q, 1, u_{0}}
$$

for all $f \in L^{r}$ with $q /(q-1) \leq r \leq \infty$.

Proposition 2.2 (see [52]). Let $2 \leq q \leq \infty, 1 \leq p \leq \infty$, u be a p-admissible weight, $\Lambda$ be a relatively separated subset of $\mathbf{R}^{d}, \Phi=\left\{\phi_{\lambda}, \lambda \in \Lambda\right\}$ be a family of functions on $\mathbf{R}^{d}, V_{2}(\Phi, \Lambda)$ be as in (2.11), and the frame operator $S$ on $V_{2}(\Phi, \Lambda)$ be defined by

$$
S f=\sum_{\lambda \in \Lambda}\left\langle f, \phi_{\lambda}\right\rangle \phi_{\lambda}, \quad f \in V_{2}(\Phi, \Lambda)
$$

Assume that $\Phi$ is a Riesz basis for $V_{2}(\Phi, \Lambda)$ and satisfies $\|\Phi\|_{q, p, u}<\infty$. Then $S^{-1} \Phi:=$ $\left\{S^{-1} \phi_{\lambda}: \lambda \in \Lambda\right\}$ is a dual Riesz basis of $\Phi, S^{-1 / 2} \Phi:=\left\{S^{-1 / 2} \phi_{\lambda}: \lambda \in \Lambda\right\}$ is an orthonormal basis of $V_{2}(\Phi, \Lambda)$, and $\left\|S^{-1} \Phi\right\|_{q, p, u}+\left\|S^{-1 / 2} \Phi\right\|_{q, p, u}<\infty$. If we further assume that $\Lambda$ is a lattice, and that the generator $\Phi$ of the space $V_{2}(\Phi, \Lambda)$ is enveloped by a function in the Wiener amalgam space $W_{\infty}\left(L_{p, u}\right)$ (hence $\|\Phi\|_{q, p, u} \leq\|\Phi\|_{\infty, p, u} \leq$ $\left.C\|h\|_{W_{\infty}\left(L_{p, u}\right)}<\infty\right)$, i.e., $\left|\phi_{\lambda}(x)\right| \leq h(x-\lambda)$ for some $h \in W_{\infty}\left(L_{p, u}\right)$, then so are dual Riesz basis $S^{-1} \Phi$ and orthonormal basis $S^{-1 / 2} \Phi$. 
Remark 2.5. Let $1 \leq p, r \leq \infty$; we say that a weight $u$ is $(p, r)$-admissible (or we say that $w(x, y):=u(x-y)$ is a $(p, r)$-admissible translation-invariant weight [51]) if there exist a weight $v$ and two positive constants $D \in(0, \infty)$ and $\theta \in(0,1)$ such that (2.15), (2.16), and

$$
\inf _{\tau>0}\|v\|_{L^{r^{\prime}}(B(\tau))}+t\left\|v u^{-1}\right\|_{L^{p^{\prime}}\left(\mathbf{R}^{d} \backslash B(\tau)\right)} \leq D t^{\theta} \quad \text { for all } t \geq 1
$$

hold, where $p^{\prime}=p /(p-1)$ and $r^{\prime}=r /(r-1)$. Clearly the $p$-admissibility of a weight agrees with its $(p, \infty)$-admissibility. Since for any weight $v$ it holds that $\|v\|_{L^{r^{\prime}}(B(\tau))} \leq$ $C\|v\|_{L^{1}(B(\tau))}$ for all $\tau \geq 1$, we then conclude that $p$-admissibility of a weight implies its $(p, r)$-admissibility for any $1 \leq r \leq \infty$.

2.5. Model. The reader may consider the following model for simplification:

(i) The generator $\Phi:=\left\{\phi_{\lambda}, \lambda \in \Lambda\right\}$ of the space $V_{r}(\Phi, \Lambda)$ is enveloped by some function $g$ in the Wiener amalgam space $W_{q}\left(L_{p, u}\right)$ with $2 \leq q \leq \infty$, i.e.,

$$
\left|\phi_{\lambda}(x)\right| \leq g(x-\lambda) \quad \text { for all } x \in \mathbf{R}^{d} \text { and } \lambda \in \Lambda \text {. }
$$

(The above envelopment assumption (2.22) for the generator $\Phi$ implies that the basic assumption (iii) $\|\Phi\|_{q, p, u}<\infty$ for the generator $\Phi$ is satisfied. The converse is true in the shift-invariant setting, $V_{r}(\Phi, \Lambda)=V_{r}\left(\phi_{1}, \ldots, \phi_{N}\right)$; particularly, the above envelopment property for the generator $\Phi$ of the space $V_{r}(\Phi, \Lambda)$ is equivalent to the basic assumption (iii) $\|\Phi\|_{q, p, u}<\infty$, and also equivalent to the property that the generators $\phi_{1}, \ldots, \phi_{N}$ of the space $V_{2}\left(\phi_{1}, \ldots, \phi_{N}\right)$ belong to the Wiener amalgam space $W_{q}\left(L_{p, u}\right)$. The envelopment assumption (2.22) for the generator $\Phi$ is not satisfied when the space $V_{q}(\Phi, \Lambda)$ is used for modeling slow-varying signals with shot noises [42].)

(ii) The average sampler $\Psi=\left\{\psi_{\gamma}: \gamma \in \Gamma\right\}$ is enveloped by some function $h$ in the Wiener amalgam space $W_{q^{*}}\left(L_{p, u}\right)$ with $q /(q-1) \leq q^{*} \leq \infty$, i.e.,

$$
\left|\psi_{\gamma}(x)\right| \leq h(x-\gamma) \text { for all } x \in \mathbf{R}^{d} \text { and } \gamma \in \Gamma \text {. }
$$

(The above envelopment assumption (2.23) for the average sampler $\Psi$ implies that the basic assumption (ii) $\|\Psi\|_{q^{*}, p, u}<\infty$ for the average sampler $\Psi$ is satisfied. The above envelopment assumption (2.23) for the average sampler $\Psi$ is not satisfied when it is a family of approximating delta functionals with variable width [28].)

(iii) The weight $u$ in the above envelopment assumptions on the generator $\Phi$ and the average sampler $\Psi$ is the polynomial weight $u_{\alpha}(x):=(1+|x|)^{\alpha}$ with $\alpha>d(1-1 / p)$ or the exponential weight $e_{D, \delta}(x):=\exp \left(D|x|^{\delta}\right)$ with $D>0$ and $\delta \in(0,1)$.

Remark 2.6. Given an exponent $p \in[1, \infty]$, a weight $u$, and two relatively separated subsets $\Gamma, \Gamma^{\prime}$ of $\mathbf{R}^{d}$, we define the matrix algebra $\mathcal{C}_{p, u}\left(\Gamma, \Gamma^{\prime}\right)$ of Sjöstrand class by

$$
\mathcal{C}_{p, u}\left(\Gamma, \Gamma^{\prime}\right):=\left\{A:=\left(A\left(\gamma, \gamma^{\prime}\right)\right)_{\gamma, \gamma^{\prime} \in \Gamma},\|A\|_{\mathcal{C}_{p, u}}<\infty\right\}
$$

where

$$
\left.\|A\|_{\mathcal{C}_{p, u}}:=\|(A u)_{*}(k)\right)_{k \in \mathbf{Z}^{d}} \|_{\ell^{p}}
$$

and

$$
(A u)_{*}(k)=\sup _{\gamma \in m+[-1 / 2,1 / 2)^{d}, \gamma^{\prime} \in m+k+[-1 / 2,1 / 2)^{d}, m \in \mathbf{Z}^{d}}\left|A\left(\gamma, \gamma^{\prime}\right) u\left(\gamma-\gamma^{\prime}\right)\right|, k \in \mathbf{Z}^{d}
$$


(see, e.g., $[8,10,27,49,51]$ ). For the case when $q=\infty, 1 \leq p \leq \infty$, and $\Gamma$ is a lattice, the envelopment property (2.23) for the average sampler $\Psi:=\left\{\psi_{\gamma}, \gamma \in \Gamma\right\}$ is characterized by $\left(\left\|\psi_{\gamma}\right\|_{L^{q}\left(\mu+[0,1]^{d}\right)}\right)_{\gamma, \mu \in \Gamma} \in \mathcal{C}_{p, u}(\Gamma, \Gamma)$. Here a subset $X$ of $\mathbf{R}^{d}$ is said to be a lattice if $1 \leq \sum_{x_{j} \in X} \chi_{x_{j}+[0,1]^{d}}(x) \leq D(X)$ for all $x \in \mathbf{R}^{d}$ and some $D(X)<\infty$.

3. Well-localized displayer. Let $1 \leq q \leq \infty$ and $V$ be a subspace of $L^{q}$. We say that $\Gamma$, a subset of $\mathbf{R}^{d}$, is a stable ideal sampling set for the space $V$ if there exist two positive constants $A, B$ such that

$$
A\|f\|_{L^{q}} \leq\left\|(f(\gamma))_{\gamma \in \Gamma}\right\|_{\ell^{q}(\Gamma)} \leq B\|f\|_{L^{q}} \text { for all } f \in V,
$$

and that $\Psi=\left\{\psi_{\gamma}: \gamma \in \Gamma\right\}$, a family of average sampling functionals, is a stable averaging sampler for the space $V$ if there exist two positive constants $A^{\prime}, B^{\prime}$ such that

$$
A^{\prime}\|f\|_{L^{q}} \leq\left\|\left(\left\langle f, \psi_{\gamma}\right\rangle\right)_{\gamma \in \Gamma}\right\|_{\ell q}(\Gamma) \leq B^{\prime}\|f\|_{L^{q}} \text { for all } f \in V
$$

(see [5]). From the above definitions of a stable ideal sampling set $\Gamma$ and a stable average sampler $\Psi$, we have that any function $f \in V$ can be reconstructed uniquely and stably from its samples $\{f(\gamma): \gamma \in \Gamma\}$ if $\Gamma$ is a stable ideal sampling set for $V$, and similarly that any function $f \in V$ can be reconstructed uniquely and stably from its average samples $\left\{\left\langle f, \psi_{\gamma}\right\rangle: \gamma \in \Gamma\right\}$ if $\Psi$ is a stable averaging sampler for $V$.

For average (ideal) sampling on the space $V_{2}(\Phi, \Lambda)$, derived from a general theorem for localized frames (see [8, Theorem 1], [24, Theorem 3.6], and [32, Theorem $13]$ ), we have the following well-localization results for its displayer (Theorems 3.1 and 3.2); see Remark 3.1 for a more general formulation of the well-localization of a displayer. From those results, it concludes that the displayer $\tilde{\Psi}$ associated with a stable (ideal) averaging sampler $\Psi$ has the same polynomial (subexponential) decay when both the average sampler $\Psi$ and the generator $\Phi$ for the space $V_{2}(\Phi, \Lambda)$ have polynomial (subexponential) decay.

TheOREM 3.1. Let $2 \leq q \leq \infty, q /(q-1) \leq q^{*} \leq \infty, 1 \leq r \leq q, 1 \leq p \leq \infty, u$ be a p-admissible weight, the subsets $\Lambda, \Gamma$ of $\mathbf{R}^{d}$ be relatively separated, the generator $\Phi=\left\{\phi_{\lambda}: \lambda \in \Lambda\right\}$ satisfy

$$
\|\Phi\|_{q, p, u}<\infty,
$$

the average sampler $\Psi=\left\{\psi_{\gamma}: \gamma \in \Gamma\right\}$ satisfy

$$
\|\Psi\|_{q^{*}, p, u}<\infty,
$$

and the space $V_{r}(\Phi, \Lambda)$ be as in (2.11). Assume that $\Phi$ is a Riesz basis of $V_{2}(\Phi, \Lambda)$ and that $\Psi$ is a stable averaging sampler for $V_{2}(\Phi, \Lambda) \subset L^{2}$. Then there exists a displayer $\tilde{\Psi}=\left\{\tilde{\psi}_{\gamma}: \gamma \in \Gamma\right\} \subset V_{1}(\Phi, \Lambda)$ such that

$$
\|\tilde{\Psi}\|_{q, p, u}<\infty,
$$

and

$$
f=\sum_{\gamma \in \Gamma}\left\langle f, \psi_{\gamma}\right\rangle \tilde{\psi}_{\gamma} \quad \text { for all } f \in V_{r}(\Phi, \Lambda) .
$$

If we further assume that $\Lambda$ is a lattice and that the generator $\Phi$ and the average sampler $\Psi$ are enveloped by some functions in the Wiener amalgam space $W_{\infty}\left(L_{p, u}\right)$, then so is the displayer $\tilde{\Psi}$. 
TheOREm 3.2. Let $1 \leq p, r \leq \infty$, u be a $p$-admissible weight, the subsets $\Lambda, \Gamma$ of $\mathbf{R}^{d}$ be relatively separated, $\Phi=\left\{\phi_{\lambda}: \lambda \in \Lambda\right\}$ be a family of continuous functions on $\mathbf{R}^{d}$ that satisfies

$$
\|\Phi\|_{\infty, p, u}<\infty
$$

and the space $V_{r}(\Phi, \Lambda)$ be as in (2.11). Assume that $\Phi$ is a Riesz basis of $V_{2}(\Phi, \Lambda)$, and that $\Gamma$ is a stable ideal sampling set for $V_{2}(\Phi, \Lambda) \subset L^{2}$. Then there exists a displayer $\tilde{\Psi}=\left\{\tilde{\psi}_{\gamma}: \gamma \in \Gamma\right\}$ such that $\tilde{\Psi} \subset V_{1}(\Phi, \Lambda),\|\tilde{\Psi}\|_{\infty, p, u}<\infty$, and

$$
f=\sum_{\gamma \in \Gamma} f(\gamma) \tilde{\psi}_{\gamma} \quad \text { for all } f \in V_{r}(\Phi, \Lambda)
$$

If we further assume that $\Gamma$ is a lattice and that $\Phi$ is enveloped by a function in the Wiener amalgam space $W_{\infty}\left(L_{p, u}\right)$, then so is the displayer $\tilde{\Psi}$.

Remark 3.1. For the well-localization of the displayer $\tilde{\Psi}$, the following general principle can be derived from theorems for localized frames (see [8, Theorem 1], [24, Theorem 3.6], and [32, Theorem 13]): Let $\Lambda$ and $\Gamma$ be two index sets, and let the families $\mathcal{A}(\Lambda)=\left\{\left(a\left(\lambda, \lambda^{\prime}\right)\right)_{\lambda, \lambda^{\prime} \in \Lambda}\right\}$ and $\mathcal{A}(\Gamma, \Lambda)=\left\{(a(\gamma, \lambda))_{\gamma \in \Gamma, \lambda \in \Lambda}\right\}$ of infinite matrices have the following algebraic properties that (i) $\mathcal{A}(\Lambda)$ is an inverse-closed matrix algebra in $\mathcal{B}\left(\ell^{2}(\Lambda)\right.$ ) (the space of all bounded operators on $\ell^{2}(\Lambda)$ ), (ii) $A^{T} A \in \mathcal{A}(\Lambda)$ for any $A \in \mathcal{A}(\Gamma, \Lambda)$, and (iii) $A B \in \mathcal{A}(\Gamma, \Lambda)$ for any $A \in \mathcal{A}(\Gamma, \Lambda)$ and $B \in \mathcal{A}(\Lambda)$. Then on the space $V_{2}(\Phi, \Lambda)$ whose generator $\Phi:=\left(\phi_{\lambda}\right)_{\lambda \in \Lambda}$ is a frame, there exists an $\mathcal{A}(\Gamma, \Lambda)$-localized displayer $\tilde{\Psi}:=\left(\tilde{\psi}_{\gamma}\right)_{\gamma \in \Gamma}$ associated with a stable average sampling processing

$$
V_{2}(\Phi, \Lambda) \ni f \longmapsto\langle f, \Psi\rangle \in \ell^{2}(\Gamma)
$$

whose average sampler $\Psi:=\left(\psi_{\gamma}\right)_{\gamma \in \Gamma}$ is $\mathcal{A}(\Gamma, \Lambda)$-localized. Here we say that $\Psi:=$ $\left(\psi_{\gamma}\right)_{\gamma \in \Gamma}$ is $\mathcal{A}(\Gamma, \Lambda)$-localized on $V_{2}(\Phi, \Lambda)$ if $A_{\Psi, \Phi}:=\left(\left\langle\psi_{\gamma}, \phi_{\lambda}\right\rangle\right)_{\gamma \in \Gamma, \lambda \in \Lambda} \in \mathcal{A}(\Gamma, \Lambda)$ and $A_{\Psi, \tilde{\Phi}}:=\left(\left\langle\psi_{\gamma}, \tilde{\phi}_{\lambda}\right\rangle\right)_{\gamma \in \Gamma, \lambda \in \Lambda} \in \mathcal{A}(\Gamma, \Lambda)$, where $\tilde{\Phi}=\left(\tilde{\phi}_{\lambda}\right)_{\lambda \in \Lambda}$ is the dual frame generator $\tilde{\Phi}$ associated with the frame $\Phi$. Thus the well-localization for the displayer in Theorems 3.1 and 3.2 becomes essentially a concrete example of the above general principle (particularly in Theorems 3.1 and 3.2, the Schur class $\mathcal{A}_{p, u}(\Gamma, \Lambda)$ and the Sjöstrand class $\mathcal{C}_{p, u}(\Gamma, \Lambda)$ are used as $\mathcal{A}(\Gamma, \Lambda)$ in the above principle, and $\mathcal{A}_{p, u}(\Lambda, \Lambda)$ and $\mathcal{C}_{p, u}(\Lambda, \Lambda)$ as $\left.\mathcal{A}(\Lambda)\right)$. The well-localization of the displayer in the above principle and the localization of the dual frame in the theory of frames are equivalent since for the case when $\Psi \in V_{2}(\Phi, \Lambda)$ (otherwise replacing $\Psi$ with the projection $P \Psi$ of $\Psi$ on $V_{2}(\Phi, \Lambda)$; see Remark 3.2 below) the stability of the average sampling process (3.9) is equivalent to the frame property for $\Psi$, and the displayer $A_{\Psi, \Phi}\left(A_{\Psi, \Phi}^{T} A_{\Psi, \Phi}\right)^{\dagger} \Phi$ associated with the average sampling processing (3.9) is the canonical dual frame associated with the frame $\Psi$, where $\left(A_{\Psi, \Phi}^{T} A_{\Psi, \Phi}\right)^{\dagger}$ is the pseudo-inverse of the matrix $A_{\Psi, \Phi}^{T} A_{\Psi, \Phi}$. Therefore the above principle for the well-localization of a displayer is established (i) in [8, Theorem 1] for the case when $\Psi=\Phi, \Gamma=\Lambda, \mathcal{A}(\Lambda, \Lambda)$, and $\mathcal{A}(\Lambda)$ are the Sjöstrand class $\mathcal{C}_{1,0}(\Lambda, \Lambda)$ defined by

$$
\mathcal{C}_{1,0}(\Lambda, \Lambda)=\left\{\left(c\left(\lambda, \lambda^{\prime}\right)_{\lambda, \lambda^{\prime} \in \Lambda}:\left(\sup _{a(\lambda)-a\left(\lambda^{\prime}\right)=g \in G}\left|c\left(\lambda, \lambda^{\prime}\right)\right|\right)_{g \in G} \in \ell^{1}(G)\right\},\right.
$$


where $G$ is an additive discrete group of the form $B_{0} \mathbf{Z}^{d} \times\left(\mathbf{Z}^{d^{\prime}} / B_{1} \mathbf{Z}^{d^{\prime}}\right)$ for some nonsingular diagonal matrices $B_{0}$ and $B_{1}$, and $a: \Lambda \rightarrow G$ is a map with $\sup _{g \in G} \#\{\lambda \in$ $\Lambda: a(\lambda)=g\}<\infty$; (ii) in [24, Theorem 3.6] for the case when $\Psi=\Phi, \Gamma=\Lambda$, $\mathcal{A}(\Lambda, \Lambda)$ and $\mathcal{A}(\Lambda)$ are a solid, inverse-closed, involute Banach algebra; and (iii) in [32, Theorem 13] for the case that $\Lambda=\mathbf{Z}^{d}, \Gamma$ is a relative separated subset of $\mathbf{R}^{d}$, $\mathcal{A}(\Lambda)=\mathcal{A}$, and $\mathcal{A}(\Gamma, \Lambda)=\left\{(a(\gamma, k))_{\gamma \in \Gamma, k \in \mathbf{Z}^{d}}:(\tilde{a}(m, n))_{m, n \in \mathbf{Z}^{d}} \in \mathcal{A}\right\}$, where $\mathcal{A}$ is a solid, inverse-closed, involute Banach algebra, and

$$
\tilde{a}(m, n)= \begin{cases}\sup _{\gamma \in\left(m+[0,1)^{d}\right) \cap \Gamma}|a(\gamma, n)| & \text { if }\left(m+[0,1)^{d}\right) \cap \Gamma \neq \emptyset, \\ 0 & \text { if }\left(m+[0,1)^{d}\right) \cap \Gamma=\emptyset .\end{cases}
$$

The above principle for the well-localization of the displayer can be derived from [32, Theorem 13] with identical proof.

Remark 3.2. Let $\Phi=\left\{\phi_{\lambda}: \lambda \in \Lambda\right\}$ and $\Psi=\left\{\psi_{\gamma}: \gamma \in \Gamma\right\}$ be as in either Theorem 3.1 or Theorem 3.2, and let $S^{-1} \Phi=\left\{S^{-1} \phi_{\lambda}: \lambda \in \Lambda\right\}$ be the dual Riesz basis for the space $V_{2}(\Phi, \Lambda)$ in Proposition 2.2. By Propositions 2.1 and 2.2, the operator $P$ defined by $\operatorname{Pf}:=\sum_{\lambda \in \Lambda}\left\langle f, S^{-1} \phi_{\lambda}\right\rangle \phi_{\lambda}$ is a projection operator from $L^{2}$ to $V_{2}(\Phi, \Lambda)$. We can extend the domain of the projection operator $P$ so that $P \psi_{\gamma}$ is well defined for every sampling functional $\psi_{\gamma}$ in the average sampling case and for the delta functional $\delta_{\gamma}$ in the ideal sampling case. Moreover, $\left\langle f, \psi_{\gamma}\right\rangle=\left\langle f, P \psi_{\gamma}\right\rangle$ for all $\gamma \in \Gamma$ and $f \in V_{2}(\Phi, \Lambda)$. We then have that if $\Psi$ is a stable average sampler, then $P \Psi=\left\{P \psi_{\gamma}: \gamma \in \Gamma\right\}$ is a frame of $V_{2}(\Phi, \Lambda)$; that is, there exist two positive constants $A, B>0$ such that

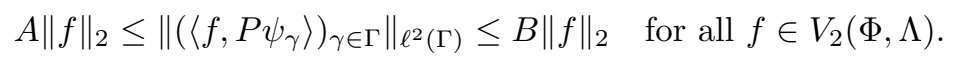

So by the general frame theory, a displayer $\tilde{\Psi} \subset V_{2}(\Phi, \Lambda)$, which may or may not have polynomial (subexponential) decay, can be constructed, while we show in Theorems 3.1 and 3.2 that a displayer $\tilde{\Psi}$ can be constructed to has polynomial (subexponential) decay whenever the generator $\Phi$ and the average sampler $\Psi$ have it. The reader may refer to [2] and references cited therein for the connection among average (ideal) sampling, reproducing kernel Hilbert space, and frame theory in the shift-invariant setting.

By Theorems 3.1 and 3.2, we have the following corollary for the uniform sampling in the familiar shift-invariant space.

Corollary 3.3. Let $1 \leq p \leq \infty, 2 \leq q \leq \infty, 1 \leq r \leq q, q /(q-1) \leq q^{*} \leq \infty$, $u$ be the polynomial weight $u_{\alpha}$ with $\alpha>d(1-1 / p)$ or the subexponential weight $e_{D, \delta}$ with $D>0$ and $\delta \in(0,1)$, and $\phi_{1}, \ldots, \phi_{N} \in W_{q}\left(L_{p, u}\right)$, and $\Phi=\left\{\phi_{n}(\cdot-k): 1 \leq n \leq\right.$ $\left.N, k \in \mathbf{Z}^{d}\right\}$ be a Riesz basis of $V_{2}\left(\phi_{1}, \ldots, \phi_{N}\right)$. Then we have that

(i) if $\psi_{1}, \ldots, \psi_{L} \in W_{q^{*}}\left(L_{p, u}\right)$, and $\Psi:=\left\{\psi_{l}(\cdot-k): 1 \leq l \leq L, k \in \mathbf{Z}^{d}\right\}$ is a stable average sampler for $V_{2}\left(\phi_{1}, \ldots, \phi_{N}\right)$, then there exist functions $\tilde{\psi}_{1}, \ldots, \tilde{\psi}_{L} \in$ $W_{q}\left(L_{p, u}\right)$ such that $f=\sum_{l=1}^{L} \sum_{k \in \mathbf{Z}^{d}}\left\langle f, \psi_{l}(\cdot-k)\right\rangle \tilde{\psi}_{l}(\cdot-k)$ holds for all $f \in$ $V_{r}\left(\phi_{1}, \ldots, \phi_{N}\right)$.

(ii) if $\phi_{1}, \ldots, \phi_{N}$ are continuous functions in $W_{\infty}\left(L_{p, u}\right)$, and $X_{0}+\mathbf{Z}^{d}$ is a stable ideal sampling set for $V_{2}\left(\phi_{1}, \ldots, \phi_{N}\right)$, where $X_{0}=\left\{x_{1}, \ldots, x_{L}\right\} \subset[0,1)^{d}$, then there exist continuous functions $\tilde{\psi}_{1}, \ldots, \tilde{\psi}_{L} \in W_{\infty}\left(L_{p, u}\right)$ such that $f=$ $\sum_{l=1}^{L} \sum_{k \in \mathbf{Z}^{d}} f\left(x_{l}+k\right) \tilde{\psi}_{l}(\cdot-k)$ holds for all $f \in V_{r}\left(\phi_{1}, \ldots, \phi_{N}\right)$.

Remark 3.3. For the shift-invariant setting $V_{2}(\Phi, \Lambda)=V_{2}\left(\phi_{1}, \ldots, \phi_{N}\right)$ for some functions $\phi_{1}, \ldots, \phi_{N}$, it is shown in [28] that if the generator $\phi_{1}, \ldots, \phi_{N}$ satisfies $\left|\phi_{n}(x)\right| \leq C_{0}(1+|x|)^{-\alpha}$ for all $1 \leq n \leq N$ and $x \in \mathbf{R}^{d}$, (i.e., $\|\Phi\|_{\infty, \infty, u_{\alpha}}<\infty$, where $\left.\Phi:=\left\{\phi_{n}(\cdot-k), 1 \leq n \leq N, k \in \mathbf{Z}^{d}\right\}\right)$, and if the average sampling functional 
$\psi_{\gamma}$ is supported in $\gamma+[-a, a]$ and $\left\|\psi_{\gamma}\right\|_{L^{1}} \leq C_{0}$ for some positive constants $C_{0}$ and $a$ (which implies that $\|\Psi\|_{1, \infty, u}<\infty$ for any weight $u$, where $\Psi:=\left\{\psi_{\gamma}, \gamma \in\right.$ $\Gamma\}$ ), then the corresponding displayer $\tilde{\Psi}:=\left\{\tilde{\psi}_{\gamma}: \gamma \in \Gamma\right\}$ can be chosen to satisfy $\left|\tilde{\psi}_{\gamma}(x)\right| \leq C_{1}(1+|x-\gamma|)^{-\alpha}$ for some positive constant $C_{1}$ (i.e., $\|\tilde{\Psi}\|_{\infty, \infty, u_{\alpha}}<\infty$ ). A similar result for the ideal sampling process is also established in [28]. The above results for the average (ideal) sampling process follow from Theorems 3.1 and 3.2 with $p=q=\infty, q^{*}=1, u=u_{\alpha}$, and $V_{2}(\Phi, \Lambda)$ being a finitely generated shift-invariant space. Other than those exponents $p, q, q^{*}$ and weight $u$ mentioned above, the results in Theorems 3.1 and 3.2 are new even for our familiar shift-invariant setting.

By Theorems 3.1 and 3.2, we have the following result for the stability of the average sampler $\Psi$ in the space $V_{r}(\Phi, \Lambda) \subset L^{r}$ with $r \neq 2$, which can also derived from [24, Theorem 2.7] and [28, Theorem 10].

Corollary 3.4. Let $2 \leq q \leq \infty, 1 \leq p \leq \infty$, u be a p-admissible weight, and the subsets $\Lambda, \Gamma$ of $\mathbf{R}^{d}$ be relatively separated. Then we have that

(i) if $\Phi$ is a Riesz basis of $V_{2}(\Phi, \Lambda)$ and satisfies (3.3), and if $\Psi$ is a stable averaging sampler for $V_{2}(\Phi, \Lambda) \subset L^{2}$ and satisfies (3.4) for some $q /(q-1) \leq$ $q^{*} \leq \infty$, then the stable average sampler $\Psi$ for $V_{2}(\Phi, \Lambda)$ is also a stable averaging sampler for $V_{r}(\Phi, \Lambda) \subset L^{r}$ for all $q^{*} /\left(q^{*}-1\right) \leq r \leq q$.

(ii) if the family $\Phi=\left\{\phi_{\lambda}: \lambda \in \Lambda\right\}$ of continuous functions on $\mathbf{R}^{d}$ is a Riesz basis of $V_{2}(\Phi, \Lambda)$ and satisfies (3.7), and if $\Gamma$ is a stable ideal sampling set for $V_{2}(\Phi, \Lambda)$, then the ideal sampling set $\Gamma$ for $V_{2}(\Phi, \Lambda)$ is also a stable ideal sampling set for $V_{r}(\Phi, \Lambda) \subset L^{r}$ for all $1 \leq r \leq \infty$.

Remark 3.4. By Corollary 3.4, the stability of the average (ideal) sampler for $V_{2}(\Phi, \Lambda) \subset L^{2}$ implies the stability of the average (ideal) sampler for $V_{r}(\Phi, \Lambda) \subset L^{r}$ with $r \neq 2$. Such an implication can also be derived from [24, Theorem 2.7] and [28, Theorem 10], where the formulation of localized frames are used. (The author thank the anonymous referee for pointing out that derivation.) The above implication is observed in $[22,28]$ for the shift-invariant setting under slightly stronger assumptions on the average sampler $\Psi$, the generator $\Phi$, and the nonuniform sampling set $\Gamma$ than the ones in Corollary 3.4. As for the case when the average sampler and the generator are identical and when the grid $\Lambda$ and the sampling set $\Gamma$ are $\mathbf{Z}^{d}$, the above implication has long been known (and even the converse is also true); see, for instance, [7, 39].

4. Stability of the average (ideal) sampling/reconstruction process. For a matrix $A:=\left(A\left(\lambda, \lambda^{\prime}\right)\right)_{\lambda \in \Lambda, \lambda \in \Lambda^{\prime}}$, we define its transpose $A^{*}$ by $A^{*}:=\left(\overline{A\left(\lambda, \lambda^{\prime}\right)}\right)_{\lambda^{\prime} \in \Lambda^{\prime}, \lambda \in \Lambda}$. For a space $V_{2}(\Phi, \Lambda)$ generated by $\Phi:=\left\{\phi_{\lambda}, \lambda \in \Lambda\right\}$, an average sampling on the space $V_{2}(\Phi, \Lambda)$ with the average sampler $\Psi:=\left\{\psi_{\gamma}, \gamma \in \Gamma\right\}$, and an ideal sampling on the space $V_{2}(\Phi, \Lambda)$ with the sampling set $\Gamma$, we define the subspace $H$ of $\ell^{2}$ by

$$
H=\left\{\left(\left\langle f, \phi_{\lambda}\right\rangle\right)_{\lambda \in \Lambda}: f \in V_{2}(\Phi, \Lambda)\right\}
$$

and two Gram matrices $A_{\Psi, \Phi}$ and $A_{\delta_{\Gamma}, \Phi}$ by

$$
A_{\Psi, \Phi}=\left(\left\langle\psi_{\gamma}, \phi_{\lambda}\right\rangle\right)_{\gamma \in \Gamma, \lambda \in \Lambda}
$$

and

$$
A_{\delta_{\Gamma}, \Phi}=\left(\phi_{\lambda}(\gamma)\right)_{\gamma \in \Gamma, \lambda \in \Lambda} .
$$

TheOREM 4.1. Let $2 \leq q \leq \infty, q /(q-1) \leq q^{*} \leq \infty ; q^{*} /\left(q^{*}-1\right) \leq r \leq q ; 1 \leq$ $p \leq \infty$; $u$ be a $p$-admissible weight; the subsets $\Lambda, \Gamma$ of $\mathbf{R}^{d}$ be relatively separated; the 
generator $\Phi=\left\{\phi_{\lambda}: \lambda \in \Lambda\right\}$ satisfy $\|\Phi\|_{q, p, u}<\infty$; the average sampler $\Psi=\left\{\psi_{\gamma}\right.$ : $\gamma \in \Gamma\}$ satisfy $\|\Psi\|_{q^{*}, p, u}<\infty$; and the space $V_{r}(\Phi, \Lambda)$, the subspace $H$ of $\ell^{2}$, and the matrix $A_{\Psi, \Phi}$ be as in (2.11), (4.1), and (4.2), respectively. Assume that $\Phi$ is a frame of $V_{2}(\Phi, \Lambda)$. Then $\Psi$ is a stable averaging sampler for $V_{2}(\Phi, \Lambda) \subset L^{2}$ if and only if there exists a positive constant $C$ such that

$$
C^{-1}\|c\|_{\ell^{2}(\Lambda)}^{2} \leq\left\langle\left(A_{\Psi, \Phi}\right)^{*} A_{\Psi, \Phi} c, c\right\rangle \leq C\|c\|_{\ell^{2}(\Lambda)}^{2} \text { for all } c \in H .
$$

TheOREM 4.2. Let $1 \leq p, r \leq \infty$; $u$ be a $p$-admissible weight; the subsets $\Lambda, \Gamma$ of $\mathbf{R}^{d}$ be relatively separated; $\Phi=\left\{\phi_{\lambda}: \lambda \in \Lambda\right\}$ be a family of continuous functions on $\mathbf{R}^{d}$ that satisfies (3.7); and the space $V_{r}(\Phi, \Lambda)$, the space $H$, and the matrix $A_{\delta_{\Gamma}, \Phi}$ be as in (2.11), (4.1), and (4.3), respectively. Assume that $\Phi$ is a frame of $V_{2}(\Phi, \Lambda)$. Then $\Gamma$ is a stable ideal sampling set for $V_{2}(\Phi, \Lambda)$ if and only if there exists a positive constant $C$ such that

$$
C^{-1}\|c\|_{\ell^{2}(\Lambda)}^{2} \leq\left\langle\left(A_{\delta_{\Gamma}, \Phi}\right)^{*} A_{\delta_{\Gamma}, \Phi} c, c\right\rangle \leq C\|c\|_{\ell^{2}(\Lambda)}^{2} \text { for all } c \in H .
$$

Remark 4.1. For the uniform average sampling on finitely generated shift-invariant spaces, that is, $V_{2}(\Phi, \Lambda)=V_{2}\left(\phi_{1}, \ldots, \phi_{N}\right)$ and $\Psi=\left\{\psi_{l}(\cdot-k), 1 \leq l \leq L, k \in \mathbf{Z}^{d}\right\}$ for some functions $\phi_{n}, 1 \leq n \leq N$, and $\psi_{l}, 1 \leq l \leq L$, the matrix $\left(A_{\Psi, \Phi}\right)^{*} A_{\Psi, \Phi}$ in (4.4) can be written as

$$
\begin{aligned}
& \left(A_{\Psi, \Phi}\right)^{*} A_{\Psi, \Phi} \\
& \quad=\left(\sum_{l=1}^{N} \sum_{j \in \mathbf{Z}^{d}}\left\langle\phi_{n}(\cdot-k), \psi_{l}(\cdot-j)\right\rangle\left\langle\psi_{l}(\cdot-j), \phi_{n^{\prime}}\left(\cdot-k^{\prime}\right)\right\rangle\right)_{(n, k),\left(n^{\prime}, k^{\prime}\right) \in\{1, \ldots, N\} \times \mathbf{Z}^{d}} .
\end{aligned}
$$

Due to the shift-invariant structure of the matrix $\left(A_{\Psi, \Phi}\right)^{*} A_{\Psi, \Phi}$, we may use the Fourier technique to interpret (4.4) in Theorem 4.1 as

$$
C^{-1} G(\xi) \leq A_{a s}(\xi) \leq C G(\xi) \text { a.e. } \xi \in \mathbf{R}^{d},
$$

where the Fourier transform $\hat{f}$ of an integrable function $f$ is defined by $\hat{f}(\xi)=$ $\int_{\mathbf{R}^{d}} f(x) e^{-i x \xi} d x$, and the $N \times N$ matrices $G(\xi)$ and $A_{a s}(\xi)$ are defined by

$$
G(\xi)=\left(\sum_{k \in \mathbf{Z}^{d}} \widehat{\phi}_{n}(\xi+2 k \pi) \overline{\widehat{\phi}_{n^{\prime}}(\xi+2 k \pi)}\right)_{1 \leq n, n^{\prime} \leq N}
$$

and

$$
A_{a s}(\xi)=\sum_{l=1}^{L} \sum_{k, k^{\prime} \in \mathbf{Z}^{d}}\left(\widehat{\phi}_{n}(\xi+2 k \pi) \overline{\widehat{\psi}_{l}(\xi+2 k \pi)} \widehat{\psi}_{l}\left(\xi+2 k^{\prime} \pi\right) \overline{\widehat{\phi}_{n^{\prime}}\left(\xi+2 k^{\prime} \pi\right)}\right)_{1 \leq n, n^{\prime} \leq N} .
$$

The above characterization (4.6) of the stable averaging sampler $\Psi$ is given in [5] under weaker assumptions on the generator $\phi_{1}, \ldots, \phi_{N}$ and the average sampler $\psi_{1}, \ldots, \psi_{L}$ than the ones for the generator $\Phi$ and the average sampler $\Psi$ in Theorem 4.1.

Remark 4.2. For the uniform ideal sampling on a single generated shift-invariant space, that is, $V_{2}(\Phi, \Lambda)=V_{2}(\phi)$ and $\Gamma=\mathbf{Z}^{d}$, the matrix $\left(A_{\delta_{\Gamma}, \Phi}\right)^{*} A_{\delta_{\Gamma}, \Phi}$ in (4.5) can be 
written as $\left(A_{\delta_{\Gamma}, \Phi}\right)^{*} A_{\delta_{\Gamma}, \Phi}=\left(\sum_{j \in \mathbf{Z}^{d}} \phi(j-k) \phi\left(j-k^{\prime}\right)\right)_{k, k^{\prime} \in \mathbf{Z}^{d}}$. Similar to the uniform average sampling case, we may use the Fourier technique to interpret (4.5) in Theorem 4.2 as follows: $C^{-1} \leq\left|\sum_{k \in \mathbf{Z}^{d}} \widehat{\phi}(\xi+2 k \pi)\right| \leq C$ for almost all $\xi \in \mathbf{R}^{d}$, which was given in $[60]$.

Remark 4.3. For the characterization of the stable average sampler and stable ideal sampling set for various spaces, there is an extensive literature (see, for instance, the recent review papers $[2,57]$ and monographs $[12,14,43]$ for ideal sampling, and $[1,2,4,5,6,28,53,54,55]$ for average sampling).

5. Robustness of the reconstruction process. For the numerical stability of the reconstruction formulas (3.6) and (3.8) when the average (ideal) sampling data and the displayer are corrupted, for instance, by the noise in the measurement, we have the following result.

TheOREM 5.1. Let $p, q, q^{*}, r, u, \Lambda, \Gamma, \Phi, \Psi, \tilde{\Psi}$ be as either in Theorem 3.1 or in Theorem 3.2. Assume that the original function $f$ belongs to $V_{r}(\Phi, \Lambda)$, and that $G=$ $\left\{g_{\gamma}: \gamma \in \Gamma\right\}$ and $\tilde{\Psi}^{\prime}=\left\{\tilde{\psi}_{\gamma}^{\prime}: \gamma \in \Gamma\right\}$ are the corrupted average sampling data and displayer, respectively. Then there exists a positive constant $C$ (independent of $f, G$, and $\tilde{\Psi}^{\prime}$ ) such that

$$
\begin{aligned}
\left\|f-\sum_{\gamma \in \Gamma} g_{\gamma} \tilde{\psi}_{\gamma}^{\prime}\right\|_{r} \leq & C\left\|\left(g_{\gamma}-\left\langle f, \psi_{\gamma}\right\rangle\right)_{\gamma \in \Gamma}\right\|_{\ell^{r}(\Gamma)}\|\tilde{\Psi}\|_{q, p, u} \\
& +C\left\|\tilde{\Psi}-\tilde{\Psi}^{\prime}\right\|_{q, p, u}\left\|\left(g_{\gamma}\right)_{\gamma \in \Gamma}\right\|_{\ell^{r}(\Gamma)} .
\end{aligned}
$$

For the numerical stability of the reconstruction processes (3.6) and (3.8) when there is certain perturbation for the average sampler and for the ideal sampling set, we have the following results.

TheOrem 5.2. Let $p, q, q^{*}, u, \Lambda, \Gamma, \Phi, \Psi, \tilde{\Psi}, V_{q}(\Phi, \Lambda)$ be as in Theorem 3.1. Then there exist a sufficiently small positive number $\delta_{0}$ and a positive constant $C$ such that any average sampler $\Psi^{\prime}=\left\{\psi_{\gamma}^{\prime}: \gamma \in \Gamma\right\}$ with the property that

$$
\left\|\Psi^{\prime}-\Psi\right\|_{q^{*}, p, u} \leq \delta_{0}
$$

is a stable average sampler for the space $V_{2}(\Phi, \Lambda)$, and the corresponding displayer $\tilde{\Psi}^{\prime}$ satisfies

$$
\left\|\tilde{\Psi}^{\prime}-\tilde{\Psi}\right\|_{q^{*}, p, u} \leq C\left\|\Psi^{\prime}-\Psi\right\|_{q^{*}, p, u} .
$$

Theorem 5.3. Let $p, u, \Lambda, \Gamma, \Phi, \tilde{\Psi}, V_{q}(\Phi, \Lambda)$ be as in Theorem 3.2. Assume that $\Phi_{\delta}=\left\{\phi_{\gamma, \delta}: \gamma \in \Gamma\right\}$ satisfies

$$
\left\|\Phi_{\delta}\right\|_{\infty, p, u} \rightarrow 0 \text { as } \delta \rightarrow 0
$$

where $\phi_{\gamma, \delta}(x)=\sup _{|t| \leq \delta}\left|\phi_{\gamma}(x+t)-\phi_{\gamma}(x)\right|$. Then there exist a sufficiently small positive number $\delta_{0}$ and a positive constant $C$ such that any sampling set $\Gamma^{\prime}:=\left\{\gamma+\delta_{\gamma}\right.$ : $\gamma \in \Gamma\}$ with $\sup _{\gamma \in \Gamma}\left|\delta_{\gamma}\right| \leq \delta_{0}$ is a stable ideal sampling set for the space $V_{2}(\Phi, \Lambda)$, and the corresponding displayer $\tilde{\Psi}^{\prime}$ satisfies $\left\|\tilde{\Psi}^{\prime}-\tilde{\Psi}\right\|_{\infty, p, u} \leq C\left\|\Phi_{\delta_{0}}\right\|_{\infty, p, u}$.

By Theorems 5.2 and 5.3, we have the following results about perturbation for nonuniform average (ideal) sampling on a finitely generated shift-invariant space.

Corollary 5.4. Let $1 \leq p \leq \infty, 2 \leq q \leq \infty, q /(q-1) \leq q^{*} \leq \infty$; $u$ be the polynomial weight $u_{\alpha}$ with $\alpha>d(1-1 / p)$ or the subexponential weight $e_{D, \delta}$ with 
$D>0$ and $\delta \in(0,1), \phi_{1}, \ldots, \phi_{N} \in W_{q}\left(L_{p, u}\right) ; \Gamma$ be a relatively separated subset of $\mathbf{R}^{d}$; and $\Phi:=\left\{\phi_{n}(\cdot-k), 1 \leq n \leq N, k \in \mathbf{Z}^{d}\right\}$ be a Riesz basis of the shift-invariant space $V_{2}\left(\phi_{1}, \ldots, \phi_{N}\right)$. Then

(i) if $\psi_{1}, \ldots, \psi_{L} \in W_{q^{*}}\left(L_{p, u}\right)$, and $\Psi:=\left\{\psi_{l}(\cdot-\gamma), 1 \leq l \leq L, \gamma \in \Gamma\right\}$ is a stable average sampler for $V_{2}\left(\phi_{1}, \ldots, \phi_{N}\right)$, then there exists a positive constant $\delta_{0}$ such that for any functions $\theta_{1}, \ldots, \theta_{L}$ with $\sum_{l=1}^{L}\left\|\psi_{l}-\theta_{l}\right\|_{W_{q *}\left(L_{p, u}\right)} \leq \delta_{0}$, their generating average sampler $\Theta:=\left\{\theta_{l}(\cdot-\gamma), 1 \leq l \leq L, \gamma \in \Gamma\right\}$ is a stable average sampler for $V_{2}\left(\phi_{1}, \ldots, \phi_{N}\right)$.

(ii) if $\phi_{1}, \ldots, \phi_{N}$ are continuous functions in $W_{\infty}\left(L_{p, u}\right)$, and $\Gamma$ is a stable ideal sampling set for $V_{2}\left(\phi_{1}, \ldots, \phi_{N}\right)$, then there exists a positive constant $\delta_{0}$ such that any relatively separated set $\tilde{\Gamma}=\left\{\gamma+\delta_{\gamma}: \gamma \in \Gamma\right\}$ with $\sup _{\gamma \in \Gamma}\left|\delta_{\gamma}\right| \leq \delta_{0}$ is a stable ideal sampling set for $V_{2}\left(\phi_{1}, \ldots, \phi_{N}\right)$.

Remark 5.1. From Corollary 5.4, we see that for the shift-invariant setting, the stability of the nonuniform average (ideal) sampling is preserved under small perturbation. Such a phenomenon is observed in $[19,41,57]$ for the ideal sampling process in the band-limited spaces and the finite-generated shift-invariant spaces, and in [4] for the average sampling process in the finitely generated shift-invariant spaces. We use a different approach than the ones in $[4,19,41,57]$ to consider the perturbation problem, and then the stability under small perturbation is shown to be preserved under weak assumptions on the generator $\Phi$ and the average sampler $\Psi$; see, for instance, [4] in which such a preservation is established only for the case when $q=\infty, q^{*}=1, p=1$, and $u=u_{0}$.

Remark 5.2. Unlike in the shift-invariant setting, the stability of the ideal sampling set is not preserved under small perturbation in our general setting, or in other words, assumption (5.4) in Theorem 5.3 cannot be eliminated if we expect that the stability of the ideal sampling set is preserved under small perturbation. For instance, let $\Lambda=\mathbf{Z}$ and $\Phi:=\left\{\phi_{k}(x):=h(2(x-k)) \cos ^{2}(4 k \pi(x-k)): k \in \mathbf{Z}\right\}$, where $h(x)=\max (1-|x|, 0)$ is the hat function. For the space $V_{2}(\Phi, \mathbf{Z})$ generated by that family of functions $\Phi$, we see that $f(k)=c(k), k \in \mathbf{Z}$, for any $f=\sum_{k \in \mathbf{Z}} c(k) \phi_{k} \in$ $V_{2}(\Phi, \mathbf{Z})$. Thus $\|f\|_{2} \leq\left\|(f(k))_{k \in \mathbf{Z}}\right\|_{\ell^{2}(\mathbf{Z})} \leq 4\|f\|_{2}$ for all $f \in V_{2}(\Phi, \mathbf{Z})$, where we have also used the facts that $\phi_{k}, k \in \mathbf{Z}$, are supported in $k+[-1 / 2,1 / 2]$ and satisfy $\frac{1}{4} \leq\left\|\phi_{k}\right\|_{2} \leq 1$. This shows that $\mathbf{Z}$ is a stable sampling set for $V_{2}(\Phi, \mathbf{Z})$. Noting that $f(k+1 /(8 k))=0, k \in \mathbf{Z}$, for any $f=\sum_{k \in \mathbf{Z}} c(k) \phi_{k} \in V_{2}(\Phi, \mathbf{Z})$, we then conclude that for any $0<\delta<1 / 2$, the small perturbation $\mathbf{Z}_{\delta}=\left\{k+(-1)^{k} \min (\delta, 1 /(8|k|)), 0 \neq k \in\right.$ $\mathbf{Z}\}$ of the stable sampling set $\mathbf{Z}$ is not a stable sampling set for $V_{2}(\Phi, \Lambda)$. Moreover, assumption (5.4) does not hold for that family of functions $\Phi$, since for any $\delta>0$,

$$
\left\|\Phi_{\delta}\right\|_{q, p, \alpha} \geq\left\|\phi_{k}-\phi_{k}\left(\cdot+\frac{1}{8 k}\right)\right\|_{L^{q}(k+[0,1])} \geq \frac{1}{2} \int_{0}^{1 / 4}|\cos 8 k \pi x| d x \geq \frac{1}{16},
$$

where the integer $k$ is chosen so that $8 k \delta \geq 1$.

6. Locally finite reconstruction process. For a bounded set $K$ and a positive number $R$, we let $B(K, R):=\left\{y \in \mathbf{R}^{d}: \inf _{x \in K}|y-x| \leq R\right\}$ be the $R$-neighborhood of the set $K$. For an average sampling process on the space $V_{r}(\Phi, \Lambda)$ with the average sampler $\Psi:=\left\{\psi_{\gamma}, \gamma \in \Gamma\right\}$, we define the locally finite reconstruction approximation of a function $f \in V_{r}(\Phi, \Lambda)$ on a bounded set $K$ using average sampling data on the $R$-neighborhood $B(K, R)$ of the set $K$ by

$$
\tilde{f}_{K, R}^{a}=\sum_{\gamma \in \Gamma \cap B(K, R)}\left\langle f, \psi_{\gamma}\right\rangle \tilde{\psi}_{\gamma, K, R}^{a}
$$


where

$$
\tilde{\psi}_{\gamma, K, R}^{a}=\sum_{\lambda_{1}, \lambda_{2} \in B(K, 2 R)}\left\langle\psi_{\gamma}, \phi_{\lambda_{1}}\right\rangle\left(\tilde{A}_{\Psi, \Phi, K, R}\right)^{-1}\left(\lambda_{1}, \lambda_{2}\right) \phi_{\lambda_{2}}
$$

and

$$
\tilde{A}_{\Psi, \Phi, K, R}=\left(\sum_{\gamma \in \Gamma \cap B(K, 4 R)}\left\langle\phi_{\lambda}, \psi_{\gamma}\right\rangle\left\langle\psi_{\gamma}, \phi_{\lambda^{\prime}}\right\rangle\right)_{\lambda, \lambda^{\prime} \in B(K, 3 R)} .
$$

Similarly for the ideal sampling process on the space $V_{r}(\Phi, \Lambda)$ with the sampling set $\Gamma$, we define the locally finite reconstruction approximation of a function $f \in V_{r}(\Phi, \Lambda)$ on a bounded set $K$ using the ideal sampling data on the $R$-neighborhood $B(K, R)$ of the set $K$ by

$$
\tilde{f}_{K, R}^{i}=\sum_{\gamma \in \Gamma \cap B(K, R)} f(\gamma) \tilde{\psi}_{\gamma, K, R}^{i}
$$

where

$$
\tilde{\psi}_{\gamma, K, R}^{i}=\sum_{\lambda_{1}, \lambda_{2} \in B(K, 2 R)} \phi_{\lambda_{1}}(\gamma)\left(\tilde{A}_{\delta_{\Gamma}, \Phi, K, R}\right)^{-1}\left(\lambda_{1}, \lambda_{2}\right) \phi_{\lambda_{2}}
$$

and

$$
\tilde{A}_{\Psi, \Phi, K, R}=\left(\sum_{\gamma \in \Gamma \cap B(K, 4 R)} \phi_{\lambda}(\gamma) \phi_{\lambda^{\prime}}(\gamma)\right)_{\lambda, \lambda^{\prime} \in B(K, 3 R)} .
$$

For any bounded set $K$, we observe that the locally finite reconstruction approximation $\tilde{f}_{K, R}^{a}$ for the average sampling/reconstruction process and $\tilde{f}_{K, R}^{i}$ for the ideal sampling/reconstruction process are obtained by using the samples in a finite neighborhood of that set $K$ with finitely many steps. Then we conclude from Theorems 6.1 and 6.2 that the locally finite reconstruction approximation could be possibly used in the real-time reconstruction by selecting the parameter $R$ properly.

Using an idea similar to the finite section method in frame theory (see, e.g., $[18,20,35])$, we have the following locally finite reconstruction approximation for the average (ideal) sampling/reconstruction process.

ThEOREM 6.1. Let $p, q, q^{*}, r, u, \Lambda, \Gamma, \Phi, \Psi, \tilde{\Psi}$ be as in Theorem 3.1, and let $\tilde{f}_{K, R}^{a}$ be defined as in (6.1) for any bounded set $K$, positive number $R \geq 1$, and function $f \in V_{r}(\Phi, \Lambda)$. Then there exists a positive constant $C$ (independent of the bounded set $K$, the positive number $R \geq 1$, and the function $f \in V_{r}(\Phi, \Lambda)$ ) such that

$$
\begin{aligned}
\left\|\tilde{f}_{K, R}^{a}-f\right\|_{L^{r}(K)} \leq & C\left\|u^{-1}\right\|_{L^{p^{\prime}}\left(\mathbf{R}^{d} \backslash B(R)\right)}\left\|\left(\left\langle f, \psi_{\gamma}\right\rangle\right)_{\gamma \in \Gamma}\right\|_{\ell^{r}(\Gamma)} \\
& +C\left\|u^{-1}\right\|_{L^{p^{\prime}}\left(\mathbf{R}^{d} \backslash B(R)\right)}\left\|\left(\left\langle f, \psi_{\gamma}\right\rangle\right)_{\gamma \in \Gamma \backslash B(K, R)}\right\|_{\ell^{r}(\Gamma \backslash B(K, R))}
\end{aligned}
$$

holds for any bounded set $K$, any positive number $R \geq 1$, and any $f \in V_{r}(\Phi, \Lambda)$.

TheOrem 6.2. Let $p, r, u, \Lambda, \Gamma, \Phi, \tilde{\Psi}$ be as in Theorem 3.2 , and let $\tilde{f}_{K, R}^{i}$ be defined as in (6.4) for any bounded set $K$, positive number $R \geq 1$, and function $f \in V_{r}(\Phi, \Lambda)$. Then there exists a positive constant $C$ (independent of the bounded set $K$, the positive number $R \geq 1$, and the function $f \in V_{r}(\Phi, \Lambda)$ ) such that

$$
\begin{aligned}
\left\|\tilde{f}_{K, R}^{i}-f\right\|_{L^{r}(K)} \leq & C\left\|u^{-1}\right\|_{L^{p^{\prime}}\left(\mathbf{R}^{d} \backslash B(R)\right)}^{2}\left\|(f(\gamma))_{\gamma \in \Gamma}\right\|_{\ell^{r}(\Gamma)} \\
& +C\left\|u^{-1}\right\|_{L^{p^{\prime}}\left(\mathbf{R}^{d} \backslash B(R)\right)}\left\|(f(\gamma))_{\gamma \in \Gamma \backslash B(K, R)}\right\|_{\ell^{r}(\Gamma \backslash B(K, R))}
\end{aligned}
$$


holds for any bounded set $K$, any positive number $R \geq 1$, and any $f \in V_{r}(\Phi, \Lambda)$.

As a by-product of Theorems 6.1 and 6.2, we have the following result about the location of (non-)ideal sampling devices.

TheOREM 6.3. Let $p, q, q^{*}, r, u, \Lambda, \Gamma, \Phi, \Psi, \tilde{\Psi}$ be as in either Theorem 3.1 or Theorem 3.2. Then there exists a positive constant $R_{0}$ such that for any bounded set $K$, the number of (non-)ideal sampling devices located in $B\left(K, R_{0}\right)$ exceeds the degrees of freedom of the space $V_{2}(\Phi, \Lambda)$ in the domain $K$, that is,

$$
\#\left(\Gamma \cap B\left(K, R_{0}\right)\right) \geq \#(\Lambda \cap K),
$$

where $\#(E)$ denotes the cardinality of a set $E$.

Remark 6.1. If $V_{2}(\Phi, \Lambda)$ is a shift-invariant space generated by a compactly supported continuous function $\phi$, then one may verify that the matrix $\left(\sum_{\gamma \in \Gamma} \phi_{\lambda}(\gamma) \phi_{\lambda^{\prime}}(\gamma)\right)$ associated with the ideal sampling on $\Gamma$ is a band-limited matrix. Furthermore for the case when finite truncation of that matrix is invertible (which is true if $\phi$ is a B-spline and $\Gamma$ is a sampling set with sampling density strictly less than the optimal density; see $[3,4])$, Gröchenig and Schwab [33] proposed an efficient local reconstruction algorithm to recover the original function in a domain exactly, instead of approximately as in Theorems 6.1 and 6.2, from its samples in a neighborhood of that domain. Comparing with the locally perfect recovery in [33], we see that the locally finite approximation in Theorems 6.1 and 6.2 works for the well-localized average sampling process as well as the ideal sampling process, and for most signals with a finite rate of innovation instead of signals of B-spline type.

Remark 6.2. The density property (6.9) in Corollary 6.3 is established in [3] for the ideal sampling of signals in a B-spline space; that is, $V_{2}(\Phi, \Lambda)$ is the shift-invariant space generated by the integer shifts of a B-spline. The reader may refer to [2] for similar results for average (ideal) sampling in the band-limited space, and to $[8,9]$ for the nonuniform Gabor system.

7. The Richardson-Landweber iterative reconstruction process. Let $\Phi$ be the generator of the space $V_{2}(\Phi, \Lambda), \Psi$ be an average sampler, and $\Gamma$ be an ideal sampling set. We define an iterative reconstruction algorithm from average sampling data $\left(a_{\gamma}\right)_{\gamma \in \Gamma} \in \ell^{r}$ by

$$
\left\{\begin{array}{l}
f_{0}=A^{-2} \sum_{\gamma \in \Gamma, \lambda \in \Lambda} a_{\gamma}\left\langle\psi_{\gamma}, \phi_{\lambda}\right\rangle \phi_{\lambda}, \\
f_{n}=f_{0}+f_{n-1}-A^{-2} T_{a s} f_{n-1} \text { if } n \geq 1,
\end{array}\right.
$$

where $A$ is a positive parameter and the operator $T_{a s}$ is defined by

$$
T_{a s} f=\sum_{\gamma \in \Gamma, \lambda \in \Lambda}\left\langle f, \psi_{\gamma}\right\rangle\left\langle\psi_{\gamma}, \phi_{\lambda}\right\rangle \phi_{\lambda} .
$$

Similarly we define an iterative reconstruction algorithm from ideal sampling data $\left(a_{\gamma}\right)_{\gamma \in \Gamma} \in \ell^{r}$ by

$$
\left\{\begin{array}{l}
f_{0}=A^{-2} \sum_{\gamma \in \Gamma, \lambda \in \Lambda} a_{\gamma} \phi_{\lambda}(\gamma) \phi_{\lambda} \\
f_{n}=f_{0}+f_{n-1}-A^{-2} T_{i s} f_{n-1}, n \geq 1
\end{array}\right.
$$

where $A$ is a positive parameter and the operator $T_{i s}$ is defined by

$$
T_{i s} f=\sum_{\gamma \in \Gamma, \lambda \in \Lambda} f(\gamma) \phi_{\lambda}(\gamma) \phi_{\lambda}
$$


Since the operators $A_{a s}$ in (7.2) and $A_{i s}$ in (7.4) can be written as $T_{a s}=$ $T_{\Phi} A_{\Psi, \Phi}^{*} A_{\Psi, \Phi}\left(T_{\Phi}\right)^{-1}$ and $T_{i s}=T_{\Phi} A_{\delta_{\Gamma}, \Phi}^{*} A_{\delta_{\Gamma}, \Phi}\left(T_{\Phi}\right)^{-1}$, where $T_{\Phi}:(c(\lambda)) \longmapsto \sum_{\lambda \in \Lambda}$ $c(\lambda) \phi_{\lambda}$, we then have that the iterative reconstruction algorithms from average sampling data and from ideal sampling data are equivalent to the familiar RichardsonLandweber iterative algorithms for the positive operators $A_{\Psi, \Phi}^{*} A_{\Psi, \Phi}$ and $A_{\delta_{\Gamma}, \Phi}^{*} A_{\delta_{\Gamma}, \Phi}$ on $\ell^{2}(\Lambda)$, respectively. For the iterative reconstruction algorithms from average sampling data and from ideal sampling data, we have the following exponential convergence for signals in $V_{r}(\Phi, \Lambda)$.

TheOREM 7.1. Let $p, q, q^{*}, r, u, \Lambda, \Gamma, \Phi, \Psi, V_{r}(\Phi, \Lambda)$ be as in Theorem 3.1. We assume that the parameter $A$ in (7.1) is a positive constant larger than the operator norm of the matrix $A_{\Psi, \Phi}$ from $\ell^{2}(\Lambda)$ to $\ell^{2}(\Gamma)$. Then the sequence $\left\{f_{n}, n \geq 1\right\}$ in (7.1) converges to a function $f_{\infty}$ in $V_{r}(\Phi, \Lambda)$ in the $L^{r}$ norm exponentially for any initial data $\left(a_{\gamma}\right)_{\gamma \in \Gamma} \in \ell^{r}$; that is, there exist two positive constants $C \in(0, \infty)$ and $s \in(0,1)$ such that

$$
\left\|f_{n}-f_{\infty}\right\|_{r} \leq C s^{n}\left\|\left(a_{\gamma}\right)_{\gamma \in \Gamma}\right\|_{\ell^{r}(\Gamma)} \quad \text { for all } n \geq 1 .
$$

Furthermore if the initial data $\left(a_{\gamma}\right)_{\gamma \in \Gamma}$ are obtained from average sampling a function $f$ in $V_{r}(\Phi, \Lambda)$, that is, $a_{\gamma}=\left\langle f, \psi_{\gamma}\right\rangle$ for all $\gamma \in \Gamma$, then the limit function $f_{\infty}$ of the sequence $\left\{f_{n}, n \geq 1\right\}$ agrees with the original function $f$.

TheOREM 7.2. Let $p, r, u, \Lambda, \Gamma, \Phi, V_{q}(\Phi, \Lambda)$ be as in Theorem 3.2. Assume that the parameter $A$ in (7.3) is a positive constant larger than the operator norm of the matrix $A_{\delta_{\Gamma}, \Phi}:=\left(\phi_{\lambda}(\gamma)\right)_{\gamma \in \Gamma, \lambda \in \Lambda}$ from $\ell^{2}(\Lambda)$ to $\ell^{2}(\Gamma)$. Then the sequence $\left\{f_{n}, n \geq 1\right\}$ in (7.3) converges to a function $f_{\infty} \in V_{r}(\Phi, \Lambda)$ in the $L^{r}$ norm exponentially. Moreover if $a_{\gamma}=f(\gamma), \gamma \in \Gamma$, holds for some $f \in V_{r}(\Phi, \Lambda)$, then $f_{\infty}=f$.

Remark 7.1. For $\Phi=\left\{\phi_{\lambda}: \lambda \in \Lambda\right\}$, we let $P$ be the projection operator from $L^{2}$ to $V_{2}(\Phi, \Lambda)$; see Remark 3.2. Then for the average sampling/reconstruction process with $\Psi$ as its average sampler (resp., the ideal sampling/reconstruction process with $\Gamma$ as its sampling set), $P \Psi:=\left\{P \psi_{\gamma}, \psi_{\gamma} \in \Psi\right\}$ (resp., $P \delta_{\Gamma}:=\left\{P \delta_{\gamma}, \gamma \in \Gamma\right\}$ ) is a frame for $V_{2}(\Phi, \Lambda)$, and hence the corresponding frame algorithm is the familiar RichardsonLandweber iterative algorithm for the positive operator $\left(A_{\Phi, \Phi}\right)^{-1 / 2} A_{\Psi, \Phi}^{*} A_{\Psi, \Phi}\left(A_{\Phi, \Phi}\right)^{-1 / 2}$ (resp., $\left(A_{\Phi, \Phi}\right)^{-1 / 2} A_{\delta_{\Gamma}, \Phi}^{*} A_{\delta_{\Gamma}, \Phi}\left(A_{\Phi, \Phi}\right)^{-1 / 2}$ ) on the space $\ell^{2}(\Lambda)$; see [2]. Clearly the iterative frame algorithm becomes the iterative algorithm proposed in this article when $\Phi$ is an orthonormal basis of $V_{2}(\Phi, \Lambda)$. In general, we need more computation for each iterative step of the iterative frame algorithm than that of the iterative algorithm in the paper. The consideration of which iterative algorithm converges faster, and of other implementations of the reconstruction process associated with the average (ideal) sampling/reconstruction process, is beyond the scope of this paper and will be discussed in a subsequent paper. By the general frame theory, the iterative frame algorithm associated with the average (ideal) sampling/reconstruction process on the space $V_{2}(\Phi, \Lambda) \subset L^{2}$ converges exponentially. Similar to the proofs of Theorems 7.1 and 7.2, we have the exponential convergence of the iterative frame algorithm on the space $V_{r}(\Phi, \Lambda)$ with $r \neq 2$, under the assumption that $p, q, q^{*}, r, u, \Lambda, \Lambda, \Phi, \Psi$ are as in Theorems 3.1 or 3.2. The above exponential convergence theorem for the iterative frame algorithm is established in $[2,22]$ for the shift-invariant setting with some minor additional assumptions on the exponents $p, q, q^{*}$ and weight $u$.

8. Proofs. In this section, we collect the proofs of all theorems and corollaries stated in Sections 3, 4, 5, 6, and 7 . 
8.1. Proof of Theorem 3.1. Unlike in the study of average (ideal) sampling signals in a shift-invariant space, the main obstacle when considering well-localization of the displayer comes from the nongroup structure on the generator $\Phi$ and the average sampler $\Psi$, which makes the standard approach from Fourier analysis inapplicable. In the proof of Theorem 3.1, we will use the procedure used in the study of localized frames (see $[8,24,31,32,37,50,51,52]$ and references cited therein) with some nonessential modification (see also Remark 3.1). For the completeness of this paper, we include a complete proof.

For a matrix $A=\left(a\left(\lambda, \lambda^{\prime}\right)\right)_{\lambda, \lambda^{\prime} \in \Lambda}$, we denote by $\|A\|_{\mathcal{B}^{2}}$ its operator norm on $\ell^{2}(\Lambda)$. To prove Theorem 3.1, we recall some properties of the matrix algebras of the Schur class $\mathcal{A}_{p, u}\left(\Lambda, \Lambda^{\prime}\right)$ and of the Sjöstrand class $\mathcal{C}_{p, u}(\Lambda, \Lambda)$ in [51]. The third property in Lemmas 8.1 and 8.2 below is usually known as Wiener's lemma; see, for instance, $[8,10,24,27,31,32,37,49,50,51]$ and references cited therein for its recent development and various applications.

Lemma 8.1 (see [51]). Let $1 \leq p \leq \tilde{p} \leq \infty$, u and $\tilde{u}$ be weights, and $\Lambda, \Lambda^{\prime}, \Lambda^{\prime \prime}$ be relatively separated subsets of $\mathbf{R}^{d}$. Then the following statements are true:

(i) If $\left\|u \tilde{u}^{-1}\right\|_{L^{r}}<\infty$, where $r=p \tilde{p} /(\tilde{p}-p)$, then

$$
\|A\|_{\mathcal{A}_{p, u}} \leq C\|A\|_{\mathcal{A}_{\tilde{p}, \tilde{u}}} \quad \text { for all } A \in \mathcal{A}_{\tilde{p}, \tilde{u}}\left(\Lambda, \Lambda^{\prime}\right) .
$$

(ii) If there exists another weight $v$ such that (2.15) and (2.16) hold, then

$$
\|A B\|_{\mathcal{A}_{p, u}} \leq C\|A\|_{\mathcal{A}_{p, u}}\|B\|_{\mathcal{A}_{p, u}}
$$

for all $A \in \mathcal{A}_{p, u}\left(\Lambda, \Lambda^{\prime}\right)$ and $B \in \mathcal{A}_{p, w}\left(\Lambda^{\prime}, \Lambda^{\prime \prime}\right)$, where

$$
A B:=\left(\sum_{\lambda^{\prime} \in \Lambda^{\prime}} A\left(\lambda, \lambda^{\prime}\right) B\left(\lambda^{\prime}, \lambda^{\prime \prime}\right)\right)_{\lambda \in \Lambda, \lambda^{\prime \prime} \in \Lambda^{\prime \prime}} .
$$

(iii) If $u$ is a p-admissible weight and $A$ is a matrix in $\mathcal{A}_{p, u}(\Lambda, \Lambda)$ satisfying

$$
\|A c\|_{\ell^{2}} \geq D_{0}\|c\|_{\ell^{2}} \text { for all } c \in \ell^{2}
$$

for some positive constant $D_{0}$, then the inverse $A^{-1}$ of the matrix $A$ belongs to $\mathcal{A}_{p, u}(\Lambda, \Lambda)$.

(iv) If $u$ is a p-admissible weight, then there exist positive constants $C_{1}, C_{2} \in$ $(0, \infty)$ and $\theta \in(0,1)$ such that the following estimate holds for all $A \in$ $\mathcal{A}_{p, u}(\Lambda, \Lambda)$ :

$$
\left\|A^{n}\right\|_{\mathcal{A}_{p, u}} \leq\left(C_{1} \frac{\|A\|_{\mathcal{A}_{p, u}}}{\|A\|_{\mathcal{B}^{2}}}\right)^{C_{2} n^{\theta}}\|A\|_{\mathcal{B}^{2}}^{n}, n \geq 1
$$

Lemma 8.2 (see [51]). Let $1 \leq p \leq \tilde{p} \leq \infty$, u and $\tilde{u}$ be weights on $\mathbf{R}^{d}$, and $\Lambda$ be a lattice of $\mathbf{R}^{d}$. Then the following statements are true:

(i) If $\left\|u \tilde{u}^{-1}\right\|_{L^{r}}<\infty$, where $r=p \tilde{p} /(\tilde{p}-p)$, then

$$
\|A\|_{\mathcal{C}_{p, u}} \leq C\|A\|_{\mathcal{C}_{\tilde{p}, \tilde{u}}} \quad \text { for all } A \in \mathcal{C}_{\tilde{p}, \tilde{u}}(\Lambda, \Lambda) .
$$

(ii) If there exists another weight $v$ such that (2.15) and (2.16) hold, then

$$
\|A B\|_{\mathcal{C}_{p, u}} \leq C\|A\|_{\mathcal{C}_{p, u}}\|B\|_{\mathcal{C}_{p, u}} \text { for all } A, B \in \mathcal{C}_{p, u}(\Lambda, \Lambda)
$$


(iii) If $u$ is a p-admissible weight on $\mathbf{R}^{d}$ and if $A$ is a matrix in $\mathcal{C}_{p, u}(\Lambda, \Lambda)$ that satisfies (8.3), then the inverse $A^{-1}$ of the matrix $A$ belong to $\mathcal{C}_{p, u}(\Lambda, \Lambda)$.

Now we are ready to start the proof of Theorem 3.1.

Proof of Theorem 3.1. By Proposition 2.2, without loss of generality, we may assume that $\Phi$ is an orthonormal basis of $V_{2}(\Phi, \Lambda)$ for otherwise replacing $\Phi$ with $S^{-1 / 2} \Phi$. Therefore

$$
\|c\|_{\ell^{2}(\Lambda)}=\left\|\sum_{\lambda \in \Lambda} c(\lambda) \phi_{\lambda}\right\|_{2} \quad \text { for all } c:=(c(\lambda))_{\lambda \in \Lambda} \in \ell^{2}(\Lambda)
$$

and

$$
f=\sum_{\lambda \in \Lambda}\left\langle f, \phi_{\lambda}\right\rangle \phi_{\lambda} \quad \text { for all } f \in V_{2}(\Phi, \Lambda)
$$

From (2.8), (3.3), and (3.4), it follows that $\|\Psi\|_{q /(q-1), p, u}+\|\Phi\|_{q, p, u}<\infty$. This, together with (2.7), Lemma 8.1, and the trivial estimate

$$
\left|A_{\Psi, \Phi}(\gamma, \lambda)\right| \leq \sum_{k \in \mathbf{Z}^{d}}\left\|\psi_{\gamma}\right\|_{L^{q /(q-1)}\left(k+[0,1]^{d}\right)}\left\|\phi_{\lambda}\right\|_{L^{q}\left(k+[0,1)^{d}\right)}
$$

proves that the matrix $A_{\Psi, \Phi}=\left(A_{\Psi, \Phi}(\gamma, \lambda)\right)_{\gamma \in \Gamma, \lambda \in \Lambda}$ in (4.2) belongs to $\mathcal{A}_{p, u}(\Gamma, \Lambda)$ :

$$
A_{\Psi, \Phi} \in \mathcal{A}_{p, u}(\Gamma, \Lambda)
$$

Furthermore, there exists a positive constant $C$ such that

$$
\left\|A_{\Psi, \Phi}\right\|_{\mathcal{A}_{p, u}} \leq C\|\Psi\|_{q /(q-1), p, u}\|\Phi\|_{q, p, u}
$$

Clearly the transpose $A^{*}$ of a matrix $A \in \mathcal{A}_{p, u}$ has the same $\mathcal{A}_{p, u}$ norm as the one of the matrix $A$,

$$
\left\|A^{*}\right\|_{\mathcal{A}_{p, u}}=\|A\|_{\mathcal{A}_{p, u}} .
$$

Combining (8.2), (8.9), and (8.11) then yields

$$
A_{\Psi, \Phi}^{*} A_{\Psi, \Phi} \in \mathcal{A}_{p, u}(\Lambda, \Lambda)
$$

For the matrix $A_{\Psi, \Phi}$, we obtain from (8.7) and the stable assumption on the averaging sampler $\Psi$ that

$$
C^{-1}\|c\|_{2} \leq\left\|\left(\sum_{\lambda \in \Lambda} A_{\Psi, \Phi}(\gamma, \lambda) c(\lambda)\right)_{\gamma \in \Gamma}\right\|_{\ell^{2}(\Gamma)} \leq C\|c\|_{2}
$$

for any $\ell^{2}(\Lambda)$-sequence $c=(c(\lambda))_{\lambda \in \Lambda}$. 
Combining (8.2), (8.12), and (8.13), and applying Lemma 8.1 to the matrix $A_{\Psi, \Phi}^{*} A_{\Psi, \Phi}$, we conclude that

$$
\left(A_{\Psi, \Phi}^{*} A_{\Psi, \Phi}\right)^{-1} \in \mathcal{A}_{p, u}(\Lambda, \Lambda) .
$$

Let

$$
R:=A_{\Psi, \Phi}\left(A_{\Psi, \Phi}^{*} A_{\Psi, \Phi}\right)^{-1},
$$

write $R=(R(\gamma, \lambda))_{\gamma \in \Gamma, \lambda \in \Lambda}$, and define $\tilde{\Psi}:=\left\{\tilde{\psi}_{\gamma}: \gamma \in \Gamma\right\}$ by

$$
\tilde{\psi}_{\gamma}=\sum_{\lambda \in \Lambda} R(\gamma, \lambda) \phi_{\lambda}, \quad \gamma \in \Gamma .
$$

Now we prove that $\tilde{\Psi}$ satisfies all requirements of the displayer associated with the average sampler $\Psi$. By (8.2), (8.9), and (8.14), we have

$$
R \in \mathcal{A}_{p, u}(\Gamma, \Lambda) .
$$

This implies that the sequence $(R(\gamma, \lambda))_{\lambda \in \Lambda} \in \ell^{1}(\Lambda)$ for any $\gamma \in \Gamma$, and hence $\tilde{\Psi} \subset$ $V_{1}(\Phi, \Lambda)$.

From (2.7), (8.2), (8.17), and the trivial estimate for $\tilde{\Psi}:\left\|\tilde{\psi}_{\gamma}\right\|_{L^{q}\left(k+[0,1]^{d}\right)} \leq$ $\sum_{\lambda \in \Lambda}|R(\gamma, \lambda)|\left\|\psi_{\lambda}\right\|_{L^{q}\left(k+[0,1]^{d}\right)}$, we have

$$
\|\tilde{\Psi}\|_{q, p, u} \leq C\|R\|_{\mathcal{A}_{p, u}}\|\Phi\|_{q, p, u}<\infty .
$$

For any $f \in V_{2}(\Phi, \lambda)$, it follows from (8.8), (8.15), and (8.17) that

$$
\begin{aligned}
& \left(\left\langle\sum_{\gamma \in \Gamma}\left\langle f, \psi_{\gamma}\right\rangle \tilde{\psi}_{\gamma}, \phi_{\lambda}\right\rangle\right)_{\lambda \in \Lambda} \\
& =\left(\sum_{\lambda_{1} \in \Lambda}\left\langle f, \phi_{\lambda_{1}}\right\rangle \times \sum_{\lambda_{2} \in \Lambda}\left\langle\phi_{\lambda_{2}}, \phi_{\lambda}\right\rangle\left(\sum_{\gamma \in \Gamma} A_{\Psi, \Phi}^{*}\left(\lambda_{1}, \gamma\right) R\left(\gamma, \lambda_{2}\right)\right)\right)_{\lambda \in \Lambda} \\
& =\left(\sum_{\lambda_{1} \in \Lambda}\left\langle f, \phi_{\lambda_{1}}\right\rangle\left\langle\phi_{\lambda_{1}}, \phi_{\lambda}\right\rangle\right)_{\lambda \in \Lambda}=\left(\left\langle f, \phi_{\lambda}\right\rangle\right)_{\lambda \in \Lambda^{.}}
\end{aligned}
$$

This proves the reconstruction formula (3.6) for $r=2$,

$$
f=\sum_{\gamma \in \Gamma}\left\langle f, \psi_{\gamma}\right\rangle \tilde{\psi}_{\gamma} \quad \text { for any } f \in V_{2}(\Phi, \Lambda) .
$$

For $1 \leq r<\infty$, we obtain from (2.8), (2.9), (8.2), (8.9), (8.17), and Proposition 2.1 that

$$
\begin{aligned}
& \left\|\sum_{\gamma \in \Gamma} \sum_{\lambda, \lambda^{\prime} \in \Lambda}|c(\lambda)| \times\left|\left\langle\phi_{\lambda}, \psi_{\gamma}\right\rangle\right| \times\left|R\left(\gamma, \lambda^{\prime}\right)\right| \times\left|\phi_{\lambda^{\prime}}\right|\right\|_{L^{r}} \\
& \quad \leq C\|c\|_{\ell^{r}(\Lambda)}\left\|A_{\Psi, \Phi}\right\|_{\mathcal{A}_{p, u}}\|R\|_{\mathcal{A}_{p, u}}\|\Phi\|_{r, p, u} \\
& \quad \leq C\|c\|_{\ell^{r}(\Lambda)}\left\|A_{\Psi, \Phi}\right\|_{\mathcal{A}_{p, u}}\|R\|_{\mathcal{A}_{p, u}}\|\Phi\|_{q, p, u}<\infty
\end{aligned}
$$

for any sequence $c:=(c(\lambda))_{\lambda \in \Lambda} \in \ell^{r}(\Lambda)$. Then for $1 \leq r<\infty$, the reconstruction formula (3.6) follows easily from (8.20), (8.21), and the density of $\ell^{2} \cap \ell^{r}$ in $\ell^{r}$. 
For $r=\infty$, we have that $p=\infty$. Take $c=(c(\lambda))_{\lambda \in \Lambda} \in \ell^{\infty}(\Lambda)$. We let $f=$ $\sum_{\lambda \in \Lambda} c(\lambda) \phi_{\lambda}$ and $f_{k, \tau}=\sum_{|\lambda-k| \leq \tau} c(\lambda) \phi_{\lambda}$ for $\tau \geq 1$. Then there exists a positive constant $C$ (independent of $k \in \mathbf{Z}^{d}$ and $\tau \geq 1$ ) such that

$$
\begin{aligned}
\sup _{x \in k+[0,1]^{d}}\left|f(x)-f_{k, \tau}(x)\right| & \leq \sum_{|\gamma-k|>\tau}|c(\lambda)| \sup _{x \in k+[0,1]^{d}}\left|\phi_{\lambda}(x)\right| \\
& \leq C\|c\|_{\ell \infty(\Lambda)}\|\Phi\|_{\infty, p, u}\left\|u^{-1}\right\|_{L^{p^{\prime}}\left(\mathbf{R}^{d} \backslash B(\tau)\right)}
\end{aligned}
$$

and

$$
\begin{aligned}
& \sup _{x \in k+[0,1]^{d}}\left|\sum_{\gamma \in \Gamma}\left\langle f-f_{k, \tau}, \psi_{\gamma}\right\rangle \tilde{\psi}_{\gamma}(x)\right| \\
& \leq\|c\|_{\ell^{\infty}}\left(\sum_{|\gamma-k| \geq \tau / 2, \gamma \in \Gamma} \sum_{\lambda \in \Lambda}+\sum_{\gamma \in \Gamma} \sum_{|\lambda-\gamma| \geq \tau / 2, \lambda \in \Lambda}\right)\left|\left\langle\phi_{\lambda}, \psi_{\gamma}\right\rangle\right| \sup _{x \in k+[0,1]^{d}}\left|\tilde{\psi}_{\gamma}(x)\right| \\
& \leq C\|c\|_{\ell^{\infty}(\Lambda)}\left\|A_{\Psi, \Phi}\right\|_{\mathcal{A}_{p, u}}\|\tilde{\Psi}\|_{\infty, p, u}\left\|u^{-1}\right\|_{L^{p^{\prime}}\left(\mathbf{R}^{d} \backslash B(\tau / 2)\right)} .
\end{aligned}
$$

The above two estimates, together with (8.20), lead to

$$
\begin{aligned}
& \sup _{x \in k+[0,1]^{d}}\left|f(x)-\sum_{\gamma \in \Gamma}\left\langle f, \psi_{\gamma}\right\rangle \tilde{\psi}_{\gamma}(x)\right| \\
& =\sup _{x \in k+[0,1]^{d}}\left|\left(f-f_{k, \tau}\right)(x)-\sum_{\gamma \in \Gamma}\left\langle f-f_{k, \tau}, \psi_{\gamma}\right\rangle \tilde{\psi}_{\gamma}(x)\right| \\
& \leq C\|c\|_{\ell^{\infty}(\Lambda)}\left\|u^{-1}\right\|_{L^{p^{\prime}}\left(\mathbf{R}^{d} \backslash B(\tau / 2)\right)} \rightarrow 0 \text { as } \tau \rightarrow \infty
\end{aligned}
$$

for all $k \in \mathbf{Z}^{d}$, where we have used assumption (2.17) to obtain the last limit. This proves the reconstruction formula (3.6) for $r=\infty$, and hence completes the verification that the collection $\tilde{\Psi}$ in (8.16) is the desired displayer associated with the average sampler $\Psi$.

Now we prove that the displayer $\tilde{\Psi}$ in (8.16) is enveloped by a function in $W_{\infty}\left(L_{p, u}\right)$ when $\Lambda$ is a lattice, and the average sampler $\Psi$ and the generator $\Phi$ for the space $V_{2}(\Phi, \Lambda)$ are enveloped by some functions $g, h \in W_{\infty}\left(L_{p, u}\right)$, respectively. Let $A_{\Psi, \Phi}=\left(A_{\Psi, \Phi}(\gamma, \lambda)\right)_{\gamma \in \Gamma, \lambda \in \Lambda}$ be as in (4.2). Then for any $\lambda \in m+[-1 / 2,1 / 2)^{d}$ and $\lambda^{\prime} \in m^{\prime}+[-1 / 2,1 / 2)^{d}$ with $m, m^{\prime} \in \mathbf{Z}^{d}$,

$$
\begin{aligned}
\left|\left(A_{\Psi, \Phi}^{*} A_{\Psi, \Phi}\right)\left(\lambda, \lambda^{\prime}\right)\right| \leq & \sum_{k, l \in \mathbf{Z}^{d}} \sum_{\mu \in \Gamma}\|h(\cdot+\lambda-k)\|_{L^{\infty}\left([0,1]^{d}\right)}\|g(\cdot+\mu-k)\|_{L^{\infty}\left([0,1]^{d}\right)} \\
& \times\|g(\cdot+\mu-l)\|_{L^{\infty}\left([0,1]^{d}\right)}\left\|h\left(\cdot+\lambda^{\prime}-l\right)\right\|_{L^{\infty}\left([0,1]^{d}\right)} \\
\leq & \sum_{k, l, n \in \mathbf{Z}^{d}}\|h(\cdot+m-k)\|_{L^{\infty}\left([-1,2]^{d}\right)}\|g(\cdot+n-k)\|_{L^{\infty}\left([-1,2]^{d}\right)} \\
& \times\|g(\cdot+n-l)\|_{L^{\infty}\left([-1,2]^{d}\right)}\left\|h\left(\cdot+m^{\prime}-l\right)\right\|_{L^{\infty}\left([-1,2]^{d}\right)} \\
\leq & d\left(m-m^{\prime}\right)
\end{aligned}
$$

for some sequence $(d(m))_{m \in \mathbf{Z}^{d}}$ with $(d(m) u(m))_{m \in \mathbf{Z}^{d}} \in \ell^{p}$. This implies that

$$
A_{\Psi, \Phi}^{*} A_{\Psi, \Phi} \in \mathcal{C}_{p, u}(\Lambda, \Lambda) .
$$


Therefore using (8.13) and (8.22), and applying Lemma 8.2 to the matrix $A_{\Psi, \Phi}^{*} A_{\Psi, \Phi}$, we conclude that

$$
\left(A_{\Psi, \Phi}^{*} A_{\Psi, \Phi}\right)^{-1} \in \mathcal{C}_{p, u}(\Lambda, \Lambda) .
$$

Let $R$ be the matrix in (8.15) and $\tilde{\Psi}:=\left\{\tilde{\psi}_{\gamma}, \gamma \in \Gamma\right\}$ be the displayer defined in (8.16). Then for $\gamma \in k+[0,1)^{d}$ and $x \in l+[0,1)^{d}$,

$$
\begin{aligned}
\left|\tilde{\psi}_{\gamma}(x)\right| \leq & \sum_{\lambda, \lambda^{\prime} \in \Lambda} \sum_{m \in \mathbf{Z}^{d}}\|g(\cdot+\gamma-m)\|_{L^{\infty}\left([0,1]^{d}\right)}\left\|h\left(\cdot+\lambda^{\prime}-m\right)\right\|_{L^{\infty}\left([0,1]^{d}\right)} \\
& \times\left|\left(A_{\Psi, \Phi}^{*} A_{\Psi, \Phi}\right)^{-1}\left(\lambda^{\prime}, \lambda\right)\right| h(x-\lambda) \\
\leq & C \sum_{n, n^{\prime}, m \in \mathbf{Z}^{d}}\|g\|_{L^{\infty}\left(m-k+[-1,2]^{d}\right)}\|h\|_{L^{\infty}\left(m-n^{\prime}+[-1,2]^{d}\right)} \\
& \times b\left(n-n^{\prime}\right) \sup _{y \in l-n+[-1,2]^{d}}|h(y)| \leq c(k-l),
\end{aligned}
$$

where $(b(k))_{k \in \mathbf{Z}^{d}}$ and $(c(k))_{k \in \mathbf{Z}^{d}}$ are sequences with $(b(k) u(k))_{k \in \mathbf{Z}^{d}}$ and $(c(k) u(k))_{k \in \mathbf{Z}^{d}}$ belonging to $\ell^{p}$. This proves that $\tilde{\Psi}:=\left\{\tilde{\psi}_{\gamma}, \gamma \in \Gamma\right\}$ is enveloped by some function in $W_{\infty}\left(L_{p, u}\right)$, and hence completes the proof.

8.2. Proof of Theorem 3.2. By (3.7) and the relative separatedness of the sets $\Gamma$ and $\Lambda$, the matrix $A_{\delta_{\Gamma}, \Phi}=\left(\phi_{\lambda}(\gamma)\right)_{\gamma \in \Gamma, \lambda \in \Lambda}$ in (4.3) belongs to $\mathcal{A}_{p, u}(\Gamma, \Lambda)$. Then we may reach the conclusion of Theorem 3.2 using the same argument as the one in the proof of Theorem 3.1, except we replace the average sampler $\Psi$ with the ideal sampler $\delta_{\Gamma}$. We omit the details of the proof here.

8.3. Proof of Theorem 4.1. Let $S$ be the frame operator (2.20) on the space $V_{2}(\Phi, \Lambda)$. By the frame assumption on the generator $\Phi$, we have

$$
C^{-1}\|f\|_{2} \leq\|S f\|_{2} \leq C\|f\|_{2} \text { for all } f \in V_{2}(\Phi, \Lambda)
$$

and

$$
C^{-1}\|f\|_{2} \leq\left\|\left(\left\langle f, \phi_{\lambda}\right\rangle\right)_{\lambda \in \Lambda}\right\|_{\ell^{2}(\Lambda)} \leq C\|f\|_{2} \quad \text { for all } f \in V_{2}(\Phi, \Lambda) .
$$

Then the conclusion follows from (8.24), (8.25), and

$$
\left\langle A_{\Psi, \Phi}^{*} A_{\Psi, \Phi} c, c\right\rangle=\left\|\left(\left\langle S f, \psi_{\gamma}\right\rangle\right)_{\gamma \in \Gamma}\right\|_{\ell^{2}(\Lambda)}^{2}
$$

for any $c=\left(\left\langle f, \phi_{\lambda}\right\rangle\right)_{\lambda \in \Lambda}$, where $f \in V_{2}(\Phi, \Lambda)$.

8.4. Proof of Theorem 4.2. Note that $\left\langle A_{\delta_{\Gamma}, \Phi}^{*} A_{\delta_{\Gamma}, \Phi} c, c\right\rangle=\left\|(S f(\gamma))_{\gamma \in \Gamma}\right\|_{\ell^{2}(\Lambda)}^{2}$ for $c=\left(\left\langle f, \phi_{\lambda}\right\rangle\right)_{\lambda \in \Lambda}$, where $f \in V_{2}(\Phi, \Lambda)$. This, together with (8.24) and (8.25), proves the conclusion.

8.5. Proof of Theorem 5.1. The estimate (5.1) follows from (3.6), (3.8), Proposition 2.1, and the following inequality:

$$
\left|f-\sum_{\gamma \in \Gamma} g_{\gamma} \tilde{\psi}_{\gamma}^{\prime}\right| \leq \sum_{\gamma \in \Gamma}\left|\left\langle f, \psi_{\gamma}\right\rangle-g_{\gamma}\right|\left|\tilde{\psi}_{\gamma}\right|+\sum_{\gamma \in \Gamma}\left|g_{\gamma}\right|\left|\tilde{\psi}_{\gamma}-\tilde{\psi}_{\gamma}^{\prime}\right| .
$$


8.6. Proof of Theorem 5.2. Let $A_{\Psi, \Phi}$ and $A_{\Psi^{\prime}, \Phi}$ be the Gram matrices as in (4.2). Then there exists a positive constant $C$ independent of the average samplers $\Psi^{\prime}$ and $\Psi$ by (2.8) and (8.10) such that

$$
\begin{aligned}
\left\|A_{\Psi, \Phi}-A_{\Psi^{\prime}, \Phi}\right\|_{\mathcal{A}_{p, u}} & =\left\|A_{\Psi-\Psi^{\prime}, \Phi}\right\|_{\mathcal{A}_{p, u}} \\
& \leq C\left\|\Psi-\Psi^{\prime}\right\|_{q /(q-1), p, \alpha}\|\Phi\|_{q, p, u} \leq C\left\|\Psi-\Psi^{\prime}\right\|_{q^{*}, p, u}\|\Psi\|_{q, p, u} .
\end{aligned}
$$

This, together with (8.2), yields the following estimate:

$$
\left\|\left(A_{\Psi, \Phi}\right)^{*} A_{\Psi, \Phi}-\left(A_{\Psi^{\prime}, \Phi}\right)^{*} A_{\Psi^{\prime}, \Phi}\right\|_{\mathcal{A}_{p, u}} \leq C\left\|\Psi-\Psi^{\prime}\right\|_{q^{*}, p, u},
$$

which then implies that

$$
\begin{aligned}
& \left\|\left(\left(A_{\Psi^{\prime}, \Phi}\right)^{*} A_{\Psi^{\prime}, \Phi}\right)^{-1}-\left(\left(A_{\Psi, \Phi}\right)^{*} A_{\Psi, \Phi}\right)^{-1}\right\|_{\mathcal{A}_{p, u}} \\
& \leq C\left\|\left(\left(A_{\Psi, \Phi}\right)^{*} A_{\Psi, \Phi}\right)^{-1}\right\|_{\mathcal{A}_{p, u}} \\
& \quad \times \sum_{k=1}^{\infty}\left\|\left(\left(\left(A_{\Psi, \Phi}\right)^{*} A_{\Psi, \Phi}\right)^{-1}\left(\left(A_{\Psi, \Phi}\right)^{*} A_{\Psi, \Phi}-\left(A_{\Psi^{\prime}, \Phi}\right)^{*} A_{\Psi^{\prime}, \Phi}\right)\right)^{k}\right\|_{\mathcal{A}_{p, u}} \\
& \leq C\left\|\left(\left(A_{\Psi, \Phi}\right)^{*} A_{\Psi, \Phi}\right)^{-1}\right\|_{\mathcal{A}_{p, u}} \\
& \quad \times \sum_{k=1}^{\infty}\left(C\left\|\left(\left(A_{\Psi, \Phi}\right)^{*} A_{\Psi, \Phi}\right)^{-1}\right\|_{\mathcal{A}_{p, u}}\left\|\left(\left(A_{\Psi, \Phi}\right)^{*} A_{\Psi, \Phi}-\left(A_{\Psi^{\prime}, \Phi}\right)^{*} A_{\Psi^{\prime}, \Phi}\right)\right\|_{\mathcal{A}_{p, u}}\right)^{k} \\
& \leq C \sum_{k=1}^{\infty}\left(C\left\|\Psi-\Psi^{\prime}\right\|_{q^{*}, p, u}\right)^{k} \leq \frac{C^{2}\left\|\Psi-\Psi^{\prime}\right\|_{q^{*}, p, u}}{1-C\left\|\Psi-\Psi^{\prime}\right\|_{q^{*}, p, u}} \leq \frac{C^{2} \delta_{0}}{1-C \delta_{0}}<\infty
\end{aligned}
$$

when $\delta_{0}$ in (5.2) is sufficiently small, where we have used the estimates in (5.2), (8.2), (8.14), and (8.27). This proves that $\left(\left(A_{\Psi^{\prime}, \Phi}\right)^{*} A_{\Psi^{\prime}, \Phi}\right)^{-1} \in \mathcal{A}_{p, u}(\Lambda, \Lambda)$ as $\delta_{0}$ in $(5.2)$ is sufficiently small. Moreover using the argument in the proof of Theorem 3.1, we conclude that $\Psi^{\prime}$ is a stable average sampler for the space $V_{2}(\Phi, \Lambda)$, and

$$
\left\|R^{\prime}-R\right\|_{p, \alpha} \leq C\left\|\Psi-\Psi^{\prime}\right\|_{q^{*}, p, \alpha}
$$

for some positive constant $C$, where $R^{\prime}=A_{\Psi^{\prime}, \Phi}\left(\left(A_{\Psi^{\prime}, \Phi}\right)^{*} A_{\Psi^{\prime}, \Phi}\right)^{-1}=\left(R^{\prime}(\gamma, \lambda)\right)_{\gamma \in \Gamma, \lambda \in \Lambda}$ and $R=A_{\Psi, \Phi}\left(\left(A_{\Psi, \Phi}\right)^{*} A_{\Psi, \Phi}\right)^{-1}=(R(\gamma, \lambda))_{\gamma \in \Gamma, \lambda \in \Lambda}$. Therefore the displayers $\tilde{\Psi}^{\prime}=$ $\left\{\tilde{\psi}_{\gamma}^{\prime}: \gamma \in \Gamma\right\}$ and $\tilde{\Psi}=\left\{\tilde{\psi}_{\gamma}: \gamma \in \Gamma\right\}$ associated with the stable average samplers $\Psi^{\prime}$ and $\Psi$, respectively, which are defined by $\psi_{\gamma}^{\prime}=\sum_{\lambda \in \Lambda} R^{\prime}(\gamma, \lambda) \phi_{\lambda}$ and $\psi_{\gamma}=$ $\sum_{\lambda \in \Lambda} R(\gamma, \lambda) \phi_{\lambda}, \gamma \in \Gamma$, satisfy

$$
\left\|\tilde{\Psi}^{\prime}-\tilde{\Psi}\right\|_{q^{*}, p, u} \leq C\left\|R-R^{\prime}\right\|_{\mathcal{A}_{p, u}}\|\Phi\|_{q^{*}, p, u} \leq C\left\|\Psi-\Psi^{\prime}\right\|_{q^{*}, p, u}
$$

Hence (5.3) follows.

8.7. Proof of Theorem 5.3. We can use the same technique as the one in the proof of Theorem 5.2, except the matrices $A_{\Psi, \Phi}$ and $A_{\Psi^{\prime}, \Phi}$ and the estimate (8.26) are replaced with the matrices $A_{\delta_{\Gamma}, \Phi}=\left(\phi_{\lambda}(\gamma)\right)_{\gamma \in \Gamma, \lambda \in \Lambda}$ and $A_{\delta_{\Gamma^{\prime}}, \Phi}=\left(\phi_{\lambda}(\gamma+\right.$ $\left.\left.\delta_{\gamma}\right)\right)_{\gamma \in \Gamma, \lambda \in \Lambda}$, and the estimate $\left\|A_{\delta_{\Gamma}, \Phi}-A_{\delta_{\Gamma^{\prime}}, \Phi}\right\|_{\mathcal{A}_{p, u}} \leq\left\|\Phi_{\delta_{0}}\right\|_{\infty, p, u}$ for sequences $\left\{\delta_{\gamma}\right\}$ with $\sup _{\gamma \in \Gamma}\left|\delta_{\gamma}\right| \leq \delta_{0}$, respectively. We omit the details of the proof here.

8.8. Proof of Corollary 5.4. The first conclusion follows from Theorem 5.2 and the equivalence between $\sum_{l=1}^{L}\left\|\theta_{n}\right\|_{W_{q^{*}}\left(L_{p, u}\right)}$ and $\|\Theta\|_{q *, p, \alpha}$, where $\Theta=\left\{\theta_{l}(\cdot-\gamma): 1 \leq\right.$ $l \leq L, \gamma \in \Gamma\}$. 
For any continuous function $\phi$ in $W_{\infty}\left(L_{p, u}\right)$, there exist continuous functions $\phi_{n}, n \geq 1$, with compact support such that $\lim _{n \rightarrow \infty}\left\|\phi_{n}-\phi\right\|_{W_{\infty}\left(L_{p, u}\right)}=0$. Therefore the modulus of continuity $\omega(\phi, \delta)(x):=\sup _{|t| \leq \delta}|\phi(x+t)-\phi(x)|$ of the continuous function $\phi$ in $W_{\infty}\left(L_{p, u}\right)$ has the property that $\|\omega(\phi, \delta)\|_{W_{\infty}\left(L_{p, u}\right)} \rightarrow 0$ as $\delta \rightarrow 0$ [2]. This together with (2.13) and Theorem 5.3 proves the second conclusion.

8.9. Proof of Theorem 6.1. For average sampling on the space $V_{r}(\Phi, \Lambda)$ with the average sampler $\Psi:=\left\{\psi_{\gamma}, \gamma \in \Gamma\right\}$, we introduce two local reconstruction approximations of a function $f \in V_{r}(\Phi, \Lambda)$ on a bounded set $K$ using average sampling data on the $R$-neighborhood $B(K, R)$ of the set $K$ by

$$
f_{K, R}=\sum_{\gamma \in \Gamma \cap B(K, R)}\left\langle f, \psi_{\gamma}\right\rangle \tilde{\psi}_{\gamma}
$$

and

$$
f_{K, R}^{1}=\sum_{\gamma \in \Gamma \cap B(K, R)}\left\langle f, \psi_{\gamma}\right\rangle \tilde{\psi}_{\gamma, K, R}^{1}
$$

where

$$
\tilde{\psi}_{\gamma}=\sum_{\lambda_{1}, \lambda_{2} \in \Lambda}\left\langle\psi_{\gamma}, \phi_{\lambda_{1}}\right\rangle\left(A_{\Psi, \Phi}^{*} A_{\Psi, \Phi}\right)^{-1}\left(\lambda_{1}, \lambda_{2}\right) \phi_{\lambda_{2}}
$$

and

$$
\tilde{\psi}_{\gamma, K, R}^{1}=\sum_{\lambda_{1}, \lambda_{2} \in B(K, 2 R) \cap \Lambda}\left\langle\psi_{\gamma}, \phi_{\lambda_{1}}\right\rangle\left(A_{\Psi, \Phi}^{*} A_{\Psi, \Phi}\right)^{-1}\left(\lambda_{1}, \lambda_{2}\right) \phi_{\lambda_{2}} .
$$

For a bounded domain $K$ and a positive number $R>0$, define the projection matrices $P_{K, R}$ and $Q_{K, R}$ by

$$
\left(P_{K, R} c\right)(\lambda)= \begin{cases}c(\lambda) & \text { if } \lambda \in \Lambda \cap B(K, R), \\ 0 & \text { if } \lambda \notin \Lambda \cap B(K, R)\end{cases}
$$

for any $c:=(c(\lambda))_{\lambda \in \Lambda}$, and

$$
\left(Q_{K, R} d\right)(\gamma)= \begin{cases}d(\gamma) & \text { if } \gamma \in \Gamma \cap B(K, R) \\ 0 & \text { if } \gamma \notin \Gamma \cap B(K, R)\end{cases}
$$

for any $d:=(d(\gamma))_{\gamma \in \Gamma}$.

To prove Theorem 6.1, we need the following estimates for $f-f_{K, R}$ and $f_{K, R}-$ $f_{K, R}^{1}$.

Lemma 8.3. Let $p, q, q^{*}, r, u, \Lambda, \Gamma, \Phi, \Psi, \tilde{\Psi}$ be as in Theorem 3.1, and set $p^{\prime}=$ $p /(p-1)$. Then there exists a positive constant $C$ (independent of the bounded set $K$, the positive number $R \geq 1$, and the function $\left.f \in V_{r}(\Phi, \Lambda)\right)$ such that

$$
\left\|f_{K, R}-f\right\|_{L^{r}(K)} \leq C\left\|u^{-1}\right\|_{L^{p^{\prime}}\left(\mathbf{R}^{d} \backslash B(R)\right)}\left\|\left(\left\langle f, \psi_{\gamma}\right\rangle\right)_{\gamma \in \Gamma \backslash B(K, R)}\right\|_{\ell^{r}(\Gamma \backslash B(K, R))}
$$

and

$$
\left\|f_{K, R}-f_{K, R}^{1}\right\|_{L^{r}(K)} \leq C\left\|u^{-1}\right\|_{L^{p^{\prime}}\left(\mathbf{R}^{d} \backslash B(R)\right)}^{2}\left\|\left(\left\langle f, \psi_{\gamma}\right\rangle\right)_{\gamma \in B(K, R)}\right\|_{\ell^{r}(B(K, R))}
$$

for any compact set $K$, any positive number $R \geq 1$, and any $f \in V_{r}(\Phi, \Lambda)$. 
Proof. Let $\tilde{K} \subset \mathbf{Z}^{d}$ be the minimal subset of $\mathbf{Z}^{d}$ such that $K \subset \tilde{K}+[0,1)^{d}$. For $1 \leq r \leq q$ and $r<\infty$,

$$
\begin{aligned}
& \left\|f-f_{K, R}\right\|_{L^{r}(K)}^{r}=\left\|\sum_{\gamma \notin \Gamma_{K, R}}\left\langle f, \psi_{\gamma}\right\rangle \tilde{\psi}_{\gamma}\right\|_{L^{r}(K)}^{r} \\
& \leq \int_{\tilde{K}+[0,1]^{d}}\left(\sum_{\gamma \notin \Gamma_{K, R}}\left|\left\langle f, \psi_{\gamma}\right\rangle\right|^{r}\left|\tilde{\psi}_{\gamma}(x)\right|\right) \times\left(\sum_{\gamma \notin \Gamma_{K, R}}\left|\tilde{\psi}_{\gamma}(x)\right|\right)^{r-1} d x \\
& \leq \sum_{\gamma \notin \Gamma_{K, R}}\left|\left\langle f, \psi_{\gamma}\right\rangle\right|^{r} \sum_{k \in \tilde{K}}\left\|\tilde{\psi}_{\gamma}\right\|_{L^{r}\left(k+[0,1]^{d}\right)} \times\left(\sum_{\gamma \notin \Gamma_{K, R}}\left\|\tilde{\psi}_{\gamma}\right\|_{L^{r}\left(k+[0,1]^{d}\right)}\right)^{r-1} \\
& \leq\left\|\left(\left\langle f, \psi_{\gamma}\right\rangle\right)_{\gamma \in \Gamma \backslash \Gamma_{K, R}}\right\|_{\ell^{r}\left(\Gamma \backslash \Gamma_{K, R}\right)}^{r} \times\left(\sup _{\gamma \notin \Gamma_{K, R}} \sum_{k \in \tilde{K}}\left\|\tilde{\phi}_{\gamma}\right\|_{L^{r}\left(k+[0,1]^{d}\right)}\right) \\
& \times\left(\sup _{k \in \tilde{K}} \sum_{\gamma \notin \Gamma_{K, R}}\left\|\tilde{\psi}_{\gamma}\right\|_{L^{r}\left(k+[0,1]^{d}\right)}\right)^{r-1}
\end{aligned}
$$

where we set $\Gamma_{K, R}=\Gamma \cap B(K, R)$. Then for all $r \in[1, \infty)$ with $1 \leq r \leq q$, we have

$$
\begin{aligned}
\left\|f-f_{K, R}\right\|_{L^{r}(K)} \leq & C\left\|\left(\left\langle f, \psi_{\gamma}\right\rangle\right)_{\gamma \in \Gamma \backslash \Gamma_{K, R}}\right\|_{\ell^{r}\left(\Gamma \backslash \Gamma_{K, R}\right)} \\
& \times\|u\|_{L^{p^{\prime}}\left(\mathbf{R}^{d} \backslash B(R)\right)}\|\tilde{\Psi}\|_{r, p, u} .
\end{aligned}
$$

For $r=\infty$, it follows from $r \leq q$ that $q=\infty$. Then using standard modification to the estimate (8.35), we obtain

$$
\begin{aligned}
\left\|f-f_{K, R}\right\|_{L^{\infty}(K)} \leq & C\left\|\left(\left\langle f, \psi_{\gamma}\right\rangle\right)_{\gamma \in \Gamma \backslash \Gamma_{K, R}}\right\|_{\ell^{\infty}\left(\Gamma \backslash \Gamma_{K, R}\right)} \\
& \times\|u\|_{L^{p^{\prime}}\left(\mathbf{R}^{d} \backslash B(R)\right)}\|\tilde{\Psi}\|_{\infty, p, u} .
\end{aligned}
$$

Then the local estimate (8.32) follows from (8.18), (8.35), and (8.36).

Set $R_{\Phi, r}=\left(\left\|\phi_{\lambda}\right\|_{L^{r}\left(k+[0,1]^{d}\right)}\right)_{\lambda \in \Lambda, k \in \mathbf{Z}^{d}}$ and $|A|=\left(\left|a_{\lambda, \lambda^{\prime}}\right|\right)$ for a matrix $A=\left(a_{\lambda, \lambda^{\prime}}\right)$. Then it follows from (2.7), (2.9), and Lemma 8.1 that

$$
\begin{aligned}
& \sup _{k \in \tilde{K}} \sum_{\gamma \in B(K, R) \cap \Gamma}\left\|\tilde{\psi}_{\gamma}-\tilde{\psi}_{\gamma, K, R}^{1}\right\|_{L^{r}\left(k+[0,1]^{d}\right)} \\
& +\sup _{\gamma \in B(K, R) \cap \Gamma} \sum_{k \in \tilde{K}}\left\|\tilde{\psi}_{\gamma}-\tilde{\psi}_{\gamma, K, R}^{1}\right\|_{L^{r}\left(k+[0,1]^{d}\right)} \\
\leq & C\left(\sup _{k \in \tilde{K}} \sum_{\gamma \in B(K, R) \cap \Gamma}+\sup _{\gamma \in B(K, R) \cap \Gamma} \sum_{k \in \tilde{K}}\right) \\
& \quad\left(\sum_{\lambda \in B(K, 2 R) \cap \Lambda} \sum_{\lambda^{\prime} \in \Lambda \backslash B(K, 2 R)}+\sum_{\lambda \in \Lambda \backslash B(K, 2 R)} \sum_{\lambda^{\prime} \in \Lambda}\right) \\
& \left|\left\langle\psi_{\gamma}, \phi_{\lambda}\right\rangle\right|\left|\left(A_{\Psi, \Phi}^{*} A_{\Psi, \Phi}\right)^{-1}\left(\lambda, \lambda^{\prime}\right)\right|\left\|\phi_{\lambda^{\prime}}\right\|_{L^{r}\left(k+[0,1]^{d}\right)} \\
\leq & C\left\|Q_{K, R}\left|A_{\Psi, \Phi}\right| P_{K, 2 R}\left|\left(A_{\Psi, \Phi}^{*} A_{\Psi, \Phi}\right)^{-1}\right|\left(I-P_{K, 2 R}\right) R_{\Phi, r} P_{K, 1}\right\|_{\mathcal{A}_{1, u}} \\
& +C\left\|Q_{K, R}\left|A_{\Psi, \Phi}\right|\left(I-P_{K, 2 R}\right)\left|\left(A_{\Psi, \Phi}^{*} A_{\Psi, \Phi}\right)^{-1}\right| R_{\Phi, r} P_{K, 1}\right\|_{\mathcal{A}_{1, u}} \\
\leq & C\|u\|_{L^{p^{\prime}}\left(\mathbf{R}^{d} \backslash B(R)\right)}^{2}\left\|\left|A_{\Psi, \Phi}\right| P_{K, 2 R}\left|\left(A_{\Psi, \Phi}^{*} A_{\Psi, \Phi}\right)^{-1}\right|\right\|_{\mathcal{A}_{p, u}}\left\|R_{\Phi, r}\right\|_{\mathcal{A}_{p, u}} \\
& +C\|u\|_{L^{p^{\prime}}\left(\mathbf{R}^{d} \backslash B(R)\right)}^{2}\left\|A_{\Psi, \Phi}\right\|_{\mathcal{A}_{p, u}}\left\|\left|\left(A_{\Psi, \Phi}^{*} A_{\Psi, \Phi}\right)^{-1}\right| R_{\Phi, r}\right\|_{\mathcal{A}_{p, u}} \\
\leq & C\|u\|_{L^{p^{\prime}}\left(\mathbf{R}^{d} \backslash B(R)\right)}^{2}\left\|A_{\Psi, \Phi}\right\|_{\mathcal{A}_{p, u}}\left\|\left(A_{\Psi, \Phi}^{*} A_{\Psi, \Phi}\right)^{-1}\right\|_{\mathcal{A}_{p, u}}\|\Phi\|_{r, p, u} .
\end{aligned}
$$


This together with (8.10) and (8.14) proves (8.33).

To prove Theorem 6.1 , we need another lemma.

LEMma 8.4. Let $p, q, q^{*}, r, u, \Lambda, \Gamma, \Phi, \Psi, \tilde{\Psi}$ be as in Theorem 3.1 , and $A_{\Psi, \Phi}$ be as in (4.2). Then there exist positive constants $C$ and $\delta_{0}$ (independent of the bounded set $K$ and the positive number $R \geq 1$ ) such that

$$
\begin{aligned}
& \left\|P_{K, 2 R}\left(A_{\Psi, \Phi}^{*} A_{\Psi, \Phi}\right)^{-1} P_{K, 2 R}-P_{K, 2 R} R_{\Psi, \Phi, K, 3 R} P_{K, 2 R}\right\|_{\mathcal{A}_{1, u_{0}}} \\
& \quad \leq C\|u\|_{L^{p^{\prime}}\left(\mathbf{R}^{d} \backslash B(R)\right)}^{2}
\end{aligned}
$$

holds for all bounded sets $K$ and all positive numbers $R$ with $\|u\|_{L^{p^{\prime}}\left(\mathbf{R}^{d} \backslash B(R)\right)} \leq \delta_{0}$, where $R_{\Psi, \Phi, K, 3 R}$ is the generalized inverse of the matrix $P_{K, 3 R} \tilde{A}_{\Psi, \Phi, K, R} P_{K, 3 R}$, that is, $R_{\Psi, \Phi, K, 3 R} P_{K, 3 R} \tilde{A}_{\Psi, \Phi, K, R} P_{K, 3 R}=P_{K, 3 R}$ and $P_{K, 3 R} R_{\Psi, \Phi, K, 3 R} P_{K, 3 R}=R_{\Psi, \Phi, K, 3 R}$.

Proof. By (8.13), for any $A \geq\left(\left\|A_{\Psi, \Phi}^{*} A_{\Psi, \Phi}\right\|_{\mathcal{B}^{2}}\right)^{1 / 2}$ there exists a matrix $B \in$ $\mathcal{A}_{p, u}(\Lambda, \Lambda)$ such that

$$
A_{\Psi, \Phi}^{*} A_{\Psi, \Phi}=A^{2}(I-B)
$$

and

$$
\|B\|_{\mathcal{B}^{2}}<1,
$$

where $I$ is the usual unit matrix. By (8.4), (8.40), and the estimates $\left\|P_{K, 3 R} B P_{K, 3 R}\right\|_{\mathcal{B}^{2}} \leq$ $\|B\|_{\mathcal{B}^{2}}$ and $\left\|P_{K, 3 R} B P_{K, 3 R}\right\|_{\mathcal{A}_{p, u}} \leq\|B\|_{\mathcal{A}_{p, u}}$, we have

$$
\left\|B^{k}\right\|_{\mathcal{A}_{p, u}}+\left\|\left(P_{K, 3 R} B P_{K, 3 R}\right)^{k}\right\|_{\mathcal{A}_{p, u}} \leq C\left(\frac{\|B\|_{\mathcal{B}^{2}}+1}{2}\right)^{k}, k \geq 1 .
$$

Therefore for $k \geq 2$,

$$
\begin{aligned}
& \quad\left\|P_{K, 2 R} \sum_{k=1}^{\infty} B^{k} P_{K, 2 R}-P_{K, 2 R} \sum_{k=1}^{\infty}\left(P_{K, 3 R} B P_{K, 3 R}\right)^{k} P_{K, 2 R}\right\|_{\mathcal{A}_{1, u_{0}}} \\
& \leq \sum_{k=2}^{\infty} \sum_{l=0}^{k-2}\left\|P_{K, 2 R}\left(P_{K, 3 R} B P_{K, 3 R}\right)^{l} B\left(I-P_{K, 3 R}\right) B^{k-1-l} P_{K, 2 R}\right\|_{\mathcal{A}_{1, u_{0}}} \\
& \leq C \sum_{k=2}^{\infty} \sum_{l=0}^{k-2}\left\|P_{K, 2 R}\left(P_{K, 3 R} B P_{K, 3 R}\right)^{l} B\left(I-P_{K, 3 R}\right)\right\|_{\mathcal{A}_{1, u_{0}}} \\
& \quad \times\left\|\left(I-P_{K, 3 R}\right) B^{k-1-l} P_{K, 2 R}\right\|_{\mathcal{A}_{1, u_{0}}} \\
& \leq C\|u\|_{L^{p^{\prime}}\left(\mathbf{R}^{d} \backslash B(R)\right)}^{2} \sum_{k=2}^{\infty} \sum_{l=0}^{k-2}\left\|\left(P_{K, 3 R} B P_{K, 3 R}\right)^{l} B\right\|_{\mathcal{A}_{p, u}}\left\|B^{k-1-l}\right\|_{\mathcal{A}_{p, u}} \\
& \leq C\|u\|_{L^{p^{\prime}}\left(\mathbf{R}^{d} \backslash B(R)\right)}^{2}\|B\|_{\mathcal{A}_{p, u}} \sum_{k=2}^{\infty}(k-1)\left(\frac{\|B\|_{\mathcal{B}^{2}}+1}{2}\right)^{k-1} \\
& \leq C\|u\|_{L^{p^{\prime}}\left(\mathbf{R}^{d} \backslash B(R)\right)}^{2},
\end{aligned}
$$

where we have used (8.40) and (8.41) to obtain the last inequality.

Write

$$
P_{K, 3 R} \tilde{A}_{\Psi, \Phi, K, R} P_{K, 3 R}=P_{K, 3 R} A_{\Psi, \Phi}^{*} Q_{K, 4 R} A_{\Psi, \Phi} P_{K, 3 R}=A^{2}\left(P_{K, 3 R}-B^{\prime}\right),
$$


where $A$ is the positive constant in (8.39). Since

$$
\begin{aligned}
& \left\|P_{K, 3 R} A_{\Psi, \Phi}^{*} Q_{K, 4 R} A_{\Psi, \Phi} P_{K, 3 R}-P_{K, 3 R} A_{\Psi, \Phi}^{*} A_{\Psi, \Phi} P_{K, 3 R}\right\|_{\mathcal{B}^{2}} \\
& \quad \leq\left\|P_{K, 3 R} A_{\Psi, \Phi}^{*} Q_{K, 4 R} A_{\Psi, \Phi} P_{K, 3 R}-P_{K, 3 R} A_{\Psi, \Phi}^{*} A_{\Psi, \Phi} P_{K, 3 R}\right\|_{\mathcal{A}_{1, u_{0}}} \\
& \quad \leq C\|u\|_{L^{p^{\prime}}\left(\mathbf{R}^{d} \backslash B(R)\right)}^{2}\left\|A_{\Psi, \Phi}\right\|_{\mathcal{A}_{p, u}}^{2},
\end{aligned}
$$

we have that

$$
\left\|P_{K, 3 R} B P_{K, 3 R}-B^{\prime}\right\|_{\mathcal{B}^{2}} \leq D_{0}\|u\|_{L^{p^{\prime}}\left(\mathbf{R}^{d} \backslash B(R)\right)}^{2}\left\|A_{\Psi, \Phi}\right\|_{\mathcal{A}_{p, u}}^{2} \leq \frac{1-\|B\|_{\mathcal{B}^{2}}}{3},
$$

when $\|u\|_{L^{p^{\prime}}\left(\mathbf{R}^{d} \backslash B(R)\right)}^{2} \leq \delta_{0}$ for some sufficiently small positive number $\delta_{0}$, where $D_{0}$ is a positive constant. Therefore

$$
\left\|\left(B^{\prime}\right)^{k}\right\|_{\mathcal{A}_{p, u}} \leq C\left(\frac{\|B\|_{\mathcal{B}^{2}}+1}{2}\right)^{k}, \quad k \geq 1
$$

by (8.4), (8.40), and (8.45). Similar to the argument in the proof of the estimate (8.42), we have

$$
\begin{aligned}
& \left\|P_{K, 2 R} \sum_{k=1}^{\infty}\left(B^{\prime}\right)^{k} P_{K, 2 R}-P_{K, 2 R} \sum_{k=1}^{\infty}\left(P_{K, 3 R} B P_{K, 3 R}\right)^{k} P_{K, 2 R}\right\|_{\mathcal{A}_{1, u}} \\
& \leq \sum_{k=1}^{\infty} \sum_{l=0}^{k-1}\left\|\left(B^{\prime}\right)^{l}\left(B^{\prime}-P_{K, 3 R} B P_{K, 3 R}\right)\left(P_{K, 3 R} B P_{K, 3 R}\right)^{k-1-l}\right\|_{\mathcal{A}_{1, u_{0}}} \\
& \leq C\|u\|_{L^{p^{\prime}}\left(\mathbf{R}^{d} \backslash B(R)\right.}^{2}\left\|A_{\Psi, \Phi}\right\|_{\mathcal{A}_{p, u}} \sum_{k=1}^{\infty} k\left(\frac{\|B\|_{\mathcal{B}^{2}}+1}{2}\right)^{k} \leq C\|u\|_{L^{p^{\prime}}\left(\mathbf{R}^{d} \backslash B(R)\right)}^{2},
\end{aligned}
$$

where the second inequality follows from (8.41), (8.44), (8.45), and (8.46). Combining (8.39), (8.42), (8.43), and (8.47) proves the desired estimate (8.38).

Now we start to prove Theorem 6.1.

Proof of Theorem 6.1. By Lemma 8.4,

$\sup _{\lambda \in B(K, 2 R)} \sum_{\lambda^{\prime} \in B(K, 2 R)}\left|\left(A_{\Psi, \Phi}^{*} A_{\Psi, \Phi}\right)^{-1}\left(\lambda, \lambda^{\prime}\right)-\left(\tilde{A}_{\Psi, \Phi, K, R}\right)^{-1}\left(\lambda, \lambda^{\prime}\right)\right| \leq C\|u\|_{L^{p^{\prime}}\left(\mathbf{R}^{d} \backslash B(R)\right)}^{2}$,

which, together with (8.9) and Lemma 8.1, implies that

$$
\begin{aligned}
& \left(\sup _{k \in \tilde{K}} \sum_{\gamma \in B(K, R) \cap \Gamma}+\sup _{\gamma \in B(K, R) \cap \Gamma} \sum_{k \in \tilde{K}}\right)\left\|\tilde{\psi}_{\gamma, K, R}^{a}-\tilde{\psi}_{\gamma, K, R}^{1}\right\|_{L^{r}\left(k+[0,1]^{d}\right)} \\
& \quad \leq C\|u\|_{L^{p^{\prime}}\left(\mathbf{R}^{d} \backslash B(R)\right)}^{2} .
\end{aligned}
$$

Therefore estimate (6.7) follows from (8.48), Proposition 2.1, and Lemma 8.3.

8.10. Proof of Theorem 6.2. Theorem 6.2 can be proved using an argument similar to the one in the proof of Theorem 6.1, except the average sampler $\Psi$ is replaced with the ideal sampler $\delta_{\Gamma}$. We omit the details of the proof here. 
8.11. Proof of Theorem 6.3. For any bounded set $K$, we let

$$
V_{2}(\Phi, \Lambda \cap K)=\left\{\sum_{\lambda \in \Lambda \cap K} c(\lambda) \phi_{\lambda}, \sum_{\lambda \in \Lambda \cap K}|c(\lambda)|^{2}<\infty\right\} .
$$

By the Riesz assumption on $\Phi$,

$$
C^{-1}\|c\|_{\ell^{2}(\Lambda \cap K)} \leq\left\|\sum_{\lambda \in \Lambda \cap K} c(\lambda) \phi_{\lambda}\right\|_{L^{2}\left(\mathbf{R}^{d}\right)} \leq C\|c\|_{\ell^{2}(\Lambda \cap K)}
$$

for any sequence $c:=(c(\lambda))_{\lambda \in \Lambda \cap K}$. For any $R \geq 1$, it follows by the localization assumption on the generator $\Phi$ that

$$
\begin{aligned}
& \left\|\sum_{\lambda \in \Lambda \cap K} c(\lambda) \phi_{\lambda}\right\|_{L^{2}\left(\mathbf{R}^{d} \backslash B(K, R)\right)}^{2} \\
& \leq \sum_{\lambda \in \Lambda \cap K}|c(\lambda)|^{2} \sum_{k \in \mathbf{Z}^{d} \backslash B(K, R-1)}\left\|\phi_{\lambda}\right\|_{L^{2}\left(k+[0,1]^{d}\right)}\left(\sum_{\lambda^{\prime} \in \Lambda \cap K}\left\|\phi_{\lambda^{\prime}}\right\|_{L^{2}\left(k+[0,1]^{d}\right)}\right) \\
& \leq C\|\Phi\|_{2, p, u}^{2}\left\|u^{-1}\right\|_{L^{p^{\prime}}\left(\mathbf{R}^{d} \backslash B(R)\right)} \sum_{\lambda \in \Lambda \cap K}|c(\lambda)|^{2} .
\end{aligned}
$$

We recall that

$$
\left\|u^{-1}\right\|_{L^{p^{\prime}}\left(\mathbf{R}^{d} \backslash B(R)\right)} \rightarrow 0 \text { as } R \rightarrow \infty
$$

by (2.17). Therefore by (8.49), (8.50), and (8.51), there exist positive constants $C$ and $R_{1}$ such that

$$
C^{-1}\|c\|_{\ell^{2}(\Lambda \cap K)} \leq\left\|\sum_{\lambda \in \Lambda \cap K} c(\lambda) \phi_{\lambda}\right\|_{L^{2}\left(B\left(K, R_{1}\right)\right)} \leq C\|c\|_{\ell^{2}(\Lambda \cap K)}
$$

for all sequences $c:=(c(\lambda))_{\lambda \in \Lambda \cap K}$.

Set $K_{1}=B\left(K, R_{1}\right)$. For the average sampling/reconstruction process, there exists a positive constant $C$ by Theorem 6.1 and Lemma 8.1 such that for any $R \geq 1$ and $f=\sum_{\lambda \in \Lambda \cap K} c(\lambda) \phi_{\lambda}$,

$$
\begin{aligned}
\left\|\tilde{f}_{K_{1}, R}^{a}-f\right\|_{L^{2}\left(K_{1}\right)} & \leq C\left\|u^{-1}\right\|_{L^{p^{\prime}}\left(\mathbf{R}^{d} \backslash B(R)\right)}\left\|\left(\left\langle f, \psi_{\gamma}\right\rangle\right)_{\gamma \in \Gamma}\right\|_{\ell^{2}(\Gamma)} \\
& \leq C\left\|u^{-1}\right\|_{L^{p^{\prime}}\left(\mathbf{R}^{d} \backslash B(R)\right)}\left\|A_{\Psi, \Phi}\right\|_{\mathcal{A}_{p, u}}\left(\sum_{\lambda \in \Lambda \cap K}|c(\lambda)|^{2}\right)^{1 / 2} .
\end{aligned}
$$

Similarly for the ideal sampling/reconstruction procedure, there exists a positive constant $C$ such that for any $R \geq 1$ and $f=\sum_{\lambda \in \Lambda \cap K} c(\lambda) \phi_{\lambda}$,

$$
\left\|\tilde{f}_{K_{1}, R}^{i}-f\right\|_{L^{2}\left(K_{1}\right)} \leq C\left\|u^{-1}\right\|_{L^{p^{\prime}}\left(\mathbf{R}^{d} \backslash B(R)\right)}\left\|A_{\delta_{\Gamma}, \Phi}\right\|_{\mathcal{A}_{p, u}}\left(\sum_{\lambda \in \Lambda \cap K}|c(\lambda)|^{2}\right)^{1 / 2} .
$$

Therefore there exists a positive constant $R_{2}$ independent of $K$ by (8.9) and (8.51)(8.54) so that

$$
C^{-1}\left(\sum_{\lambda \in \Lambda \cap K}|c(\lambda)|^{2}\right)^{1 / 2} \leq\left\|f_{K_{1}, R_{2}}^{a}\right\|_{L^{2}\left(K_{1}\right)} \leq C\left(\sum_{\lambda \in \Lambda \cap K}|c(\lambda)|^{2}\right)^{1 / 2}
$$


for the average sampling/reconstruction process, and

$$
C^{-1}\left(\sum_{\lambda \in \Lambda \cap K}|c(\lambda)|^{2}\right)^{1 / 2} \leq\left\|f_{K_{1}, R_{2}}^{i}\right\|_{L^{2}\left(K_{1}\right)} \leq C\left(\sum_{\lambda \in \Lambda \cap K}|c(\lambda)|^{2}\right)^{1 / 2}
$$

for the ideal sampling/reconstruction process. Therefore conclusion (6.9) follows by letting $R_{0}=R_{1}+R_{2}$.

8.12. Proof of Theorem 7.1. Let $A_{\Psi, \Phi}$ be as in (4.2), and the matrix $B \in$ $\mathcal{A}_{p, u}(\Lambda, \Lambda)$ be as in (8.39) and (8.40). Write the sequence $\left(a_{\gamma}\right)_{\gamma \in \Gamma}$ as a vector, to be denoted by $a$, and the family of functions $\Phi$ as a vector, which is still denoted by $\Phi$. We claim that

$$
f_{n}=\sum_{k=0}^{n} a^{T} A_{\Psi, \Phi} B^{k} \Phi, \quad n \geq 0,
$$

where $a^{T}$ denotes the transpose of the vector $a$. The above claim is obviously true for $n=0$. Inductively we assume that the claim is true for $n$. By (7.1), (8.39), and the inductive hypothesis, we have

$$
\begin{aligned}
f_{n+1} & =a^{T} A_{\Psi, \Phi} \Phi+\sum_{k=0}^{n} a^{T} A_{\Psi, \Phi} B^{k} \Phi-A^{-2} \sum_{k=0}^{n} a^{T} A_{\Psi, \Phi} B^{k} A_{\Psi, \Phi}^{*} A_{\Psi, \Phi} \Phi \\
& =a^{T} A_{\Psi, \Phi} \Phi+\sum_{k=0}^{n} a^{T} A_{\Psi, \Phi} B^{k} \Phi-\sum_{k=0}^{n} a^{T} A_{\Psi, \Phi} B^{k}(I-B) \Phi \\
& =\sum_{k=0}^{n+1} a^{T} A_{\Psi, \Phi} B^{k} \Phi .
\end{aligned}
$$

This proves claim (8.57) by induction.

By (8.9), (8.41), (8.57), and Proposition 2.1, we have

$$
\begin{aligned}
\left\|f_{n+1}-f_{n}\right\|_{r} & =\left\|a^{T} A_{\Psi, \Phi} B^{n+1} \Phi\right\|_{r} \\
& \leq C\|a\|_{\ell^{r}(\Gamma)}\left\|A_{\Psi, \Phi}\right\|_{\mathcal{A}_{p, u}}\left\|B^{n+1}\right\|_{\mathcal{A}_{p, u}}\|\Phi\|_{q, p, u} \\
& \leq C\left(\frac{\|B\|_{\mathcal{B}^{2}}+1}{2}\right)^{n}\|a\|_{\ell^{r}(\Gamma)} \quad \text { for all } n \geq 0,
\end{aligned}
$$

where $a:=\left(a_{\gamma}\right)_{\gamma \in \Gamma}$. The first conclusion then follows from (8.58).

Now we assume that the initial data $a:=\left(a_{\gamma}\right)_{\gamma \in \Gamma}$ are obtained from average sampling a function $f \in V_{r}(\Phi, \Lambda)$. Taking limits at both sides of $f_{n}=f_{0}+f_{n-1}-$ $A^{-2} T_{a s} f_{n-1}$ and using the Riesz property of $\Phi$, we obtain

$$
\sum_{\gamma \in \Gamma}\left\langle f-f_{\infty}, \psi_{\gamma}\right\rangle\left\langle\psi_{\gamma}, \phi_{\lambda}\right\rangle=0 \text { for all } \lambda \in \Lambda \text {. }
$$

Write

$$
f-f_{\infty}=\sum_{\lambda \in \Lambda} d_{\lambda} \phi_{\lambda}
$$

for some $\ell^{r}$ sequence $d=\left(d_{\lambda}\right)_{\lambda \in \Lambda}$. We then may write (8.59) as

$$
d^{T} A_{\Psi, \Phi}^{*} A_{\Psi, \Phi}=0 .
$$

Combining (8.13), (8.60), and (8.61) leads to the second conclusion of the theorem that the limit function $f_{\infty}$ agrees with the original function $f$. 
8.13. Proof of Theorem 7.2. We may use the same argument as in the proof of Theorem 7.1 with standard modification, for instance, the matrix $A_{\Psi, \Phi}$ in the proof of Theorem 7.1 by the matrix $A_{\delta_{\Gamma}, \Phi}:=\left(\phi_{\lambda}(\gamma)\right)_{\gamma \in \Gamma, \lambda \in \Lambda}$. We omit the details of the proof here.

Acknowledgments. The author would like to thank Professors A. Aldroubi, K. Gröchenig, and D. Han for their help in preparing this paper. The author also thanks the anonymous referees for their valuable comments and suggestions which led to an improvement of the results and the presentation in the paper. In particular, the author thanks the anonymous referee for the suggestion that leads to the general principle in Remark 3.1 for the well-localization of the displayer, and for pointing out that the results in Theorems 3.1 and 3.2 can be derived from theorems for localized frames in $[8,24,32]$.

\section{REFERENCES}

[1] A. Aldroubi, Non-uniform weighted average sampling and reconstruction in shift-invariant and wavelet spaces, Appl. Comput. Harmon. Anal., 13 (2002), pp. 151-161.

[2] A. Aldroubi And K. Gröchenig, Nonuniform sampling and reconstruction in shift-invariant spaces, SIAM Rev., 43 (2001), pp. 585-620.

[3] A. Aldroubi and K. Gröchenig, Beurling-Landau-type theorems for non-uniform sampling in shift invariant spaces, J. Fourier Anal. Appl., 6 (2000), pp. 93-103.

[4] A. Aldroubi AND I. Krishtal, Robustness of sampling and reconstruction and BeurlingLandau-type theorems for shift invariant spaces, Appl. Comput. Harmon. Anal., 20 (2006), pp. 250-260.

[5] A. Aldroubi, Q. Sun, And W.-S. TAng, Convolution, average sampling and a Calderon resolution of the identity for shift-invariant spaces, J. Fourier Anal. Appl., 11 (2005), pp. 215244.

[6] A. Aldroubi, Q. Sun, And W.-S. TANG, Non-uniform average sampling and reconstruction in multiply generated shift-invariant spaces, Constr. Approx., 20 (2004), pp. 173-189.

[7] A. Aldroubi, Q. Sun, And W.-S. Tang, p-frames and shift invariant subspaces of $L^{p}$, J. Fourier Anal. Appl., 7 (2001), pp. 1-21.

[8] R. Balan, P. G. Casazza, C. Heil, and Z. Landau, Density, overcompleteness and localization of frames I: theory, J. Fourier Anal. Appl., 12 (2006), pp. 105-143.

[9] R. Balan, P. G. Casazza, C. Heil, and Z. Landau, Density, overcompeleteness and localization of frames II: Gabor System, J. Fourier Anal. Appl., 12 (2006), pp. 309-344.

[10] A. G. BASKAKOV, Wiener's theorem and asymptotic estimates for elements in inverse matrices, Funktsional Anal. i. Prilozhen., 24 (1990), pp. 64-65.

[11] E. Beller AND G. DE HAAN, New algorithms for motion estimation on interlaced video, in Visual Communication and Image Processing, 3309, 1998, pp. 111-121.

[12] J. J. Benedetto And P. J. S. G. Ferreira, eds., Modern Sampling Theory: Mathematics and Applications, Birkhäuser Boston, Boston, MA, 2001.

[13] J. J. Benedetto AND S. LI, The theory of multiresolution analysis frames and applications to filter banks, Appl. Comput. Harmon. Anal., 5 (1998), pp. 389-427.

[14] J. J. Benedetto And A. I. Zayed, eds., Sampling, Wavelets, and Tomography, Birkhäuser Boston, Boston, MA, 2003.

[15] T. Blu, P. ThÉvenaz, And M. Unser, Linear interpolation revitalized, IEEE Trans. Image Process., 13 (2004), pp. 710-719.

[16] M. Bownik, The structure of shift-invariant subspaces of $L^{2}\left(R^{n}\right)$, J. Funct. Anal., 177 (2000), pp. 282-309.

[17] P. G. Casazza, The art of frame theory, Taiwanese J. Math., 4 (2000), pp. 129-201.

[18] P. G. Casazza and O. Christensen, Approximation of the inverse frame operator and applications to Gabor frames, J. Approx. Theory, 103 (2000), pp. 338-356.

[19] W. Chen, S. Itoh, And J. Shiki, Irregular sampling theorems for wavelet subspaces, IEEE Trans. Inform. Theory, 44 (1998), pp. 1131-1142.

[20] O. Christensen And T. Strohmer, The finite section method and problems in frame theory, J. Approx. Theory, 133 (2005), pp. 221-237.

[21] C. K. Chui And Q. Sun, Affine frame decompositions and shift-invariant spaces, Appl. Comput. Harmon. Anal., 20 (2006), pp. 74-107. 
[22] E. Cordero and K. Gröchenig, Localization of frames II, Appl. Comput. Harmonic Anal., 17 (2004), pp. 29-47.

[23] R. J.-M. Cramer, R. A. Scholtz, And M. Z. Win, Evaluation of an ultra wide-band propagation channel, IEEE Trans. Antennas and Propagation, 50 (2002), pp. 561-569.

[24] M. Fornasier and K. Gröchenig, Intrinsic localization of frames, Constr. Approx., 22 (2005), pp. 395-415.

[25] H. G. Feichtinger And K. Gröchenig, Irregular sampling theorems and series expansions of band-limited functions, J. Math. Anal. Appl., 167 (1992), pp. 530-556.

[26] K. GröcheniG, Foundation of Time-Frequency Analysis, Birkhäuser Boston, Boston, MA, 2001.

[27] K. Gröchenig, Time-frequency analysis of Sjöstrand class, Rev. Mat. Iberoamericana, 22 (2006), pp. 703-724.

[28] K. GröchenIG, Localization of frames, Banach frames, and the invertibility of the frame operator, J. Fourier Anal. Appl., 10 (2004), pp. 105-132.

[29] K. Gröchenig, Acceleration of the frame algorithm, IEEE Trans. Signal Process., 41 (1993), pp. 3331-3340.

[30] K. GröcheniG, Reconstruction algorithms in irregular sampling, Math. Comp., 59 (1992), pp. 181-194.

[31] K. Gröchenig and M. Leinert, Wiener's lemma for twisted convolution and Gabor frames, J. Amer. Math. Soc., 17 (2003), pp. 1-18.

[32] K. GRÖChenig AND M. LEINERT, Symmetry of matrix algebras and symbolic calculus for infinite matrices, Trans. Amer. Math. Soc., 358 (2006), pp. 2695-2711.

[33] K. Gröchenig And H. Schwab, Fast local reconstruction methods for nonuniform sampling in shift-invariant spaces, SIAM J. Matrix Anal. Appl., 24 (2003), pp. 899-913.

[34] Y. Hao, P. Marziliano, M. Vetterli, and T. Blu, Sampling and reconstruction of ECG as a signal with a finite rate of innovation, IEEE Trans. Biomedical Engineering, submitted.

[35] J. A. Hogan, Frame-based non-uniform sampling in Paley-Wiener spaces, J. Fourier Anal. Appl., to appear.

[36] H. S. Hou And H. C. Andrews, Cubic splines for image interpolation and digital filtering, IEEE Trans. Acoust. Speech Signal Process., 26 (1978), pp. 508-517.

[37] S. Jaffard, Properiétés des matrices bien localisées prés de leur diagonale et quelques applications, Ann. Inst. H. Poincaré, 7 (1990), pp. 461-476.

[38] A. J. E. M. Janssen, Duality and biorthogonality for Weyl-Heisenberg frames, J. Fourier Anal. Appl., 1 (1995), pp. 403-436.

[39] R.-Q. JiA AND C. A. MicchelLI, Using the refinement equations for the construction of prewavelets. II. Powers of two, in Curves and Surfaces (Chamonix-Mont-Blanc, 1990), Academic Press, Boston, MA, 1991, pp. 209-246.

[40] J. Kusuma, I. Maravic, and M. Vetterli, Sampling with finite rate of innovation: Channel and timing estimatation for UWB and GPS, in Proceedings of the IEEE Conference on Communication, Anchorage, AK, 2003, pp. 3540-3544.

[41] Y. LiU, Irregular sampling for spline wavelet subspaces, IEEE Trans. Inform. Theory, 42 (1996), pp. 623-627.

[42] I. Maravic And M. Vetterli, Sampling and reconstruction of signals with finite rate of innovation in the presence of noise, IEEE Trans. Signal Process., 53 (2005), pp. 2788-2805.

[43] F. A. Marvasti, ED., Nonuniform Sampling: Theory and Practice, Inf. Technol. Transm., Process. Storage, Kluwer Academic/Plenum Publishers, New York, 2001.

[44] P. Marziliano and M. Vetterli, Reconstruction of irregular sampled discrete-time bandlimited signals with unknown sampling locations, IEEE Trans. Signal Process., 48 (2000), pp. 3462-3471.

[45] P. Marziliano, M. Vetterli, And T. Blu, Sampling and exact reconstruction of bandlimited signals with shot noise, IEEE Trans. Inform. Theory, 52 (2006), pp. 2230-2233.

[46] P. Marziliano, Sampling Innovations, Ph.D. thesis, Swiss Federal Institute of Technology, Zurich, Switzerland, 2001.

[47] A. Ron And Z. Shen, Weyl-Heisenberg frames and Riesz bases in $L_{2}\left(R^{d}\right)$, Duke Math. J., 89 (1997), pp. 237-282.

[48] L. L. Schumaker, Spline Functions: Basic Theory, John Wiley \& Sons, New York, 1981.

[49] J. SJöstrand, An algebra of pseudodifferential operators, Math. Res. Lett., 1 (1994), pp. 185192.

[50] Q. Sun, Wiener's lemma for infinite matrices with polynomial off-diagonal decay, C. R. Math. Acad. Sci. Paris, 340 (2005), pp. 567-570.

[51] Q. Sun, Wiener's lemma for infinite matrices with polynomial and subexponential off-diagonal decay, Trans. Amer. Math. Soc., to appear. 
[52] Q. Sun, Frame in spaces with finite rate of innovation, Adv. Comput. Math., http://www.springerlink.com/content/rxg9553u61641005 (2006).

[53] W. Sun AND X. ZHOU, Average sampling in shift invariant subspaces with symmetric averaging functions, J. Math. Anal. Appl., 287 (2003), pp. 279-295.

[54] W. Sun And X. Zhou, Reconstruction of band-limited functions from local averages, Constr. Approx., 18 (2002), pp. 205-222.

[55] W. Sun And X. Zhou, Average sampling theorems for shift-invariant subspaces, Sci. China Ser. E 43 (2000), pp. 524-530.

[56] G. Thomas, A comparison of motion-compensated interlace-to-progressive conversion method, Image Communication, 12 (1998), pp. 209-229.

[57] M. Unser, Sampling - 50 years after Shannon, Proc. IEEE, 88 (2000), pp. 569-587.

[58] M. Vetterli, P. Marziliano, and T. Blu, Sampling signals with finite rate of innovation, IEEE Trans. Signal Process., 50 (2002), pp. 1417-1428.

[59] R. H. Vollmerhausen and R. G. Driggers, Analysis of Sampled Imaging Systems, SPIE Press, Bellingham, WA, 2000.

[60] G. G. WALter, A sampling theorem for wavelet subspaces, IEEE Trans. Inform. Theory, 38 (1992), pp. 881-884.

[61] R. G. WILEY, Recovery of bandlimited signals from unequally spaced samples, IEEE Trans. Comm., 26 (1978), pp. 135-137. 